Novos métodos incrementais para otimização convexa não-diferenciável em dois níveis com aplicações em reconstrução de imagens em tomografia por emissão 



\section{Novos métodos incrementais para otimização convexa não-diferenciável em dois níveis com aplicações em reconstrução de imagens em tomografia por emissão}

\section{Lucas Eduardo Azevedo Simões ${ }^{1}$}

Orientador: Prof. Dr. Elias Salomão Helou Neto

Dissertação apresentada ao Instituto de Ciências Matemáticas e de Computação - ICMC-USP, como parte dos requisitos para obtenção do título de Mestre em Ciências - Ciências de Computação e Matemática Computacional. VERSÃO REVISADA

USP - São Carlos

Maio de 2013

\footnotetext{
${ }^{1}$ O aluno foi financiado pela FAPESP (Processo: 2011/02219-4).
} 
Ficha catalográfica elaborada pela Biblioteca Prof. Achille Bassi e Seção Técnica de Informática, ICMC/USP, com os dados fornecidos pelo(a) autor(a)

\begin{tabular}{|c|c|}
\hline \multirow[t]{3}{*}{$\mathrm{S} 614 \mathrm{n}$} & $\begin{array}{l}\text { Simões, Lucas Eduardo Azevedo } \\
\quad \text { Novos métodos incrementais para otimização convexa } \\
\text { não-diferenciável em dois níveis com aplicaçoses em } \\
\text { reconstrução de imagens em tomografia por emissão / } \\
\text { Lucas Eduardo Azevedo Simões; orientador Elias } \\
\text { Salomão Helou. -- São Carlos, } 2013 \text {. } \\
\quad 56 \text { p. }\end{array}$ \\
\hline & $\begin{array}{l}\text { Dissertação (Mestrado - Programa de Pós-Graduação en } \\
\text { Ciências de Computação e Matemática Computacional) -- } \\
\text { Instituto de Ciências Matemáticas e de Computação, } \\
\text { Universidade de São Paulo, } 2013 \text {. }\end{array}$ \\
\hline & $\begin{array}{l}\text { 1. otimização convexa. 2. otimização não- } \\
\text { diferenciável. 3. otimização em dois níveis. } 4 . \\
\text { algoritmos incrementais. I. Helou, Elias Salomão, } \\
\text { orient. II. Título. }\end{array}$ \\
\hline
\end{tabular}




\section{Agradecimentos}

Agradeço primeiramente a Deus pela sabedoria que me foi concedida durante todo este período de trabalho, aos meus pais, Eduardo e Sandra, juntamente com a minha família, por sempre acreditarem e me apoiarem em todos os meus sonhos, a minha amada companheira Francielle, por trilhar junto comigo o mesmo caminho, ao meu orientador Elias, por ter me dado suporte indispensável durante toda esta etapa, aos meus amigos, Francys e Victor, por tornarem este trajeto um pouco mais descontraído e alegre, e por fim, à agência FAPESP pelo apoio financeiro. 

"Os grandes navegantes só são grandes por causa das grandes tempestades." 



\section{Conteúdo}

1 Introdução $\quad$ p. 1

1.1 Otimização Convexa . . . . . . . . . . . . . . . p. 1

1.2 Objetivo e Contribuições . . . . . . . . . . . . . . p. 5

2 Novos Métodos de Minimização em Dois Níveis $\quad$ p.7

2.1 Resultados Preliminares, Definições e Notações . . . . . . . . . . . . . . . . p. 7

2.2 Algoritmo I . . . . . . . . . . . . . . . . p. 12

2.2 .1 Propriedades . . . . . . . . . . . . . . p. 12

2.2.2 Resultados Principais . . . . . . . . . . . . . . p. 13

2.3 Algoritmo II . . . . . . . . . . . . . . . . . . . p. 19

2.4 Relação com Métodos Existentes . . . . . . . . . . . . . . . . p. 25

2.5 Operadores de Otimalidade Aplicáveis . . . . . . . . . . . . p. 26

2.6 Um Operador de Factibilidade Aplicável . . . . . . . . . . . . . . . . p. 28

3 Tomografia e Métodos de Reconstrução de Imagens p.33

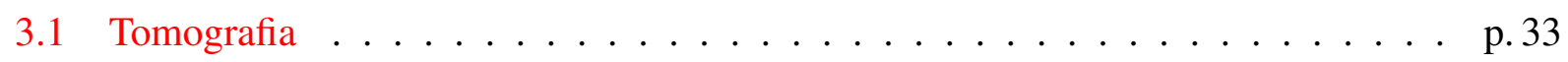

3.1 .1 Tomografia por Transmissão . . . . . . . . . . . . . . p. 33

3.1 .2 Tomografia por Emissão . . . . . . . . . . . . . . p. 34

3.1.3 Modelagem Matemática Contínua . . . . . . . . . . . . . p. 35

3.1.4 Modelagem Matemática Discreta . . . . . . . . . . . . . p. 37

3.2 Métodos . . . . . . . . . . . . . . . . . p. 38

3.2.1 Técnica de Reconstrução Algébrica - ART . . . . . . . . . . . . . p. 39

3.2.2 Projeção sobre Convexos - POCS . . . . . . . . . . . . p. p. 40 


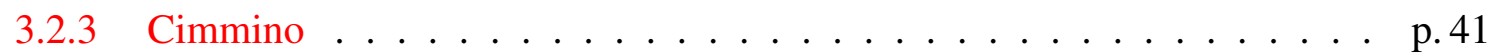

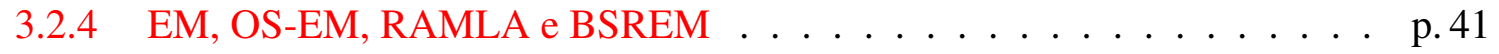

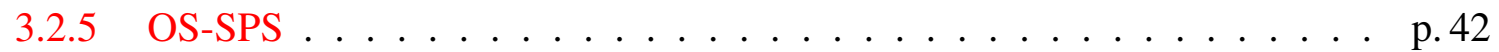

3.2.6 Um Método Alternativo de Reconstrução Tomográfica . . . . . . . . . p. 43

4 Resultados Numéricos p. 45

4.1 Problema Idealizado . . . . . . . . . . . . . . . . . p. 45

4.2 Problema de Recuperação de Imagem ． . . . . . . . . . . . . . . . . . p.49

5 Conclusão $\quad$ p. 52

$\begin{array}{ll}\text { Bibliografia } & \text { p.53 }\end{array}$ 


\section{Resumo}

Apresentamos dois novos métodos para a solução de problemas de otimização convexa em dois níveis não necessariamente diferenciáveis, i.e., mostramos que as sequências geradas por ambos os métodos convergem para o conjunto ótimo de uma função não suave sujeito a um conjunto que também envolve a minimização de uma função não diferenciável. Ambos os algoritmos dispensam qualquer tipo de resolução de subproblemas ou busca linear durante suas iterações. Ao final, para demonstrar que os métodos são viáveis, resolvemos um problema de reconstrução de imagens tomográficas. 



\begin{abstract}
We present two new methods for solving bilevel convex optimization problems, where both functions are not necessarily differentiable, i.e., we show that the sequences generated by those methods converge to the optimal set of a nonsmooth function subject to a set that also involves a function minimization. Both algorithms do not require any kind of subproblems resolution or linear search during the iterations. At the end, to prove that our methods are viable, we solve a problem of tomographic image reconstruction.
\end{abstract}





\section{$1 \quad$ Introdução}

Apresentamos neste trabalho métodos para a solução de problemas de otimização convexa em dois níveis não necessariamente diferenciáveis, permitindo a inclusão de algoritmos incrementais durante suas iterações.

Mais especificamente, neste capítulo expomos uma breve introdução sobre os métodos mais conhecidos para a solução de problemas de otimização convexa não necessariamente diferenciáveis e discutimos os algoritmos mais recentes desenvolvidos para a solução de problemas que envolvam minimização convexa em dois níveis. Ao final, discorremos sobre o objetivo e contribuição da nossa pesquisa.

\subsection{Otimização Convexa}

Sejam $f: \mathbb{R}^{n} \rightarrow \mathbb{R}$ uma função convexa não necessariamente diferenciável e $X \subset \mathbb{R}^{n}$ um conjunto convexo e fechado. Consideremos o problema de encontrar $\bar{x} \in \mathbb{R}^{n}$ que satisfaça

$$
\bar{x} \in \arg \min _{x \in X} f(x) .
$$

Dada a importância deste tipo de problema na literatura científica, muitos estudos vem sendo realizados com o objetivo de desenvolver métodos para solucioná-lo. Um importante conceito para a aplicação destas pesquisas é a generalização do gradiente para uma função convexa:

Definição 1.1 Dada uma função convexa $f: \mathbb{R}^{n} \rightarrow \mathbb{R}$ e $x \in \mathbb{R}^{n}$, definimos o subdiferencial $\partial f(x)$ de f em x como

$$
\partial f(x):=\left\{v \in \mathbb{R}^{n} \mid f(x)+\langle v, y-x\rangle \leq f(y), \quad \forall y \in \mathbb{R}^{n}\right\} .
$$

Um elemento pertencente ao conjunto $\partial f(x)$ é dito ser um subgradiente de f em $x$.

Como consequência desta definição, observa-se que o subdiferencial de uma função convexa em um ponto arbitrário $x$ no interior de seu domínio é sempre não-vazio e possui um único elemento se, e só se, a função é diferenciável em $x$. Mais ainda, este único elemento é exatamente o gradiente 
da função em $x$ (ver [1]).

Encontramos em [2] um dos primeiros algoritmos desenvolvido para a solução de (1.1) quando $X=\mathbb{R}^{n}$, chamado de Método do Subgradiente. Essencialmente, o algoritmo consiste em "andar" no sentido oposto ao subgradiente com um determinado tamanho de passo, todavia, ao contrário do método de máxima descida, não há a garantia de decrescimento da função objetivo a cada iteração. O método não depende de nenhuma busca linear para a sua convergência, bastando que os tamanhos de passo satisfaçam as seguintes regras:

$$
\lambda_{k} \geq 0, \quad \lambda_{k} \rightarrow 0 \quad \text { e } \quad \sum_{k=1}^{\infty} \lambda_{k}=\infty .
$$

Em outras palavras, uma iteração $k$ deste algoritmo pode ser descrita como

$$
x_{k+1}=x_{k}-\lambda_{k} v_{k},
$$

sendo $v_{k} \in \partial f\left(x_{k}\right)$ e $\lambda_{k}$ representando o $k$-ésimo tamanho de passo. Alternativamente, para o caso em que $X \neq \mathbb{R}^{n}$, mas é um conjunto simples o suficiente para que a projeção sobre ele não seja custosa, pode-se fazer uso do seguinte algoritmo:

$$
x_{k+1}=\mathscr{P}_{X}\left(x_{k}-\lambda_{k} v_{k}\right)
$$

Métodos mais eficientes foram desenvolvidos quando a função custo $f$ é representada como a soma de várias funções convexas $f_{i}$, ou seja, quando

$$
f(x)=\sum_{i=1}^{m} f_{i}(x)
$$

Como exemplo, podemos citar o Método de Subgradiente Incremental. Apresentamos aqui duas versões deste método, sendo a primeira [3] desenvolvida para um função objetivo Lipschitz contínua

$$
\begin{aligned}
x_{k, 0} & :=x_{k} \\
x_{k, i} & :=x_{k, i-1}-\lambda_{k} v_{k, i}, \quad i=1, \ldots, m, \quad v_{k, i} \in \partial f_{i}\left(x_{k, i-1}\right) \\
x_{k+1} & :=\mathscr{P}_{X}\left(x_{k, m}\right)
\end{aligned}
$$

e a segunda proposta por Nedić e Bertsekas [4], a qual exige que $f$ seja convexa mas não necessariamente diferenciável

$$
\begin{aligned}
x_{k, 0} & :=x_{k} \\
x_{k, i} & :=\mathscr{P}_{X}\left(x_{k, i-1}-\lambda_{k} v_{k, i}\right), \quad i=1, \ldots, m, \quad v_{k, i} \in \partial f_{i}\left(x_{k, i-1}\right) \\
x_{k+1} & :=x_{k, m} .
\end{aligned}
$$


Por fim, exibimos o Método do Subgradiente Incremental Agregado [5] como alternativa aos métodos anteriores:

$$
\begin{aligned}
x_{k, 0} & :=x_{k} \\
x_{k, i} & :=\mathscr{P}_{X}\left(x_{k, i-1}-\frac{\lambda_{k}}{m} \bar{v}_{k, i}\right), \quad i=1, \ldots, m \\
x_{k+1} & :=x_{k, m},
\end{aligned}
$$

sendo $\bar{v}_{k, i} \in \partial f_{i}\left(x_{k, i-1}\right) \operatorname{com} \bar{v}_{k, i}:=\bar{v}_{k, i-1}-v_{k-1, i}+v_{k, i}$ e $\bar{v}_{k, 0}=\sum_{i=1}^{m} v_{k, 1}$.

Ainda, para problemas de larga escala, podemos encontrar na literatura o estudo desenvolvido por Bertsekas [6]. Dada uma função custo que pode ser representada por $f(x)=\sum_{i=1}^{m}\left(s_{i}(x)+h_{i}(x)\right)$, sendo $s_{i}$ funções apropriadas para iterações proximais e $h_{i}$ funções adequadas para iterações de subgradientes, o referido trabalho garante que os seguintes algoritmos convergem para a solução ótima de (1.1):

Algoritmo Proximal 1

$$
\begin{aligned}
z_{k, i} & =\arg \min _{x \in X}\left\{s_{i}(x)+\frac{1}{2 \alpha_{k}}\left\|x-x_{k, i-1}\right\|^{2}\right\} \quad i=1, \ldots, m ; \\
x_{k, i} & =\mathscr{P}_{X}\left(z_{k}-\alpha_{k} \tilde{\nabla} h_{i}\left(z_{k}\right)\right) \quad i=1, \ldots, m ;
\end{aligned}
$$

Algoritmo Proximal 2

$$
\begin{aligned}
& z_{k, i}=\arg \min _{x \in \mathbb{R}^{n}}\left\{s_{i}(x)+\frac{1}{2 \alpha_{k}}\left\|x-x_{k, i-1}\right\|^{2}\right\} \quad i=1, \ldots, m ; \\
& x_{k, i}=\mathscr{P}_{X}\left(z_{k}-\alpha_{k} \tilde{\nabla} h_{i}\left(z_{k}\right)\right) \quad i=1, \ldots, m ;
\end{aligned}
$$

Algoritmo Proximal 3

$$
\begin{aligned}
& z_{k, i}=x_{k, i-1}-\alpha_{k} \tilde{\nabla} h_{i}\left(x_{k, i-1}\right) \quad i=1, \ldots, m \\
& x_{k, i}=\arg \min _{x \in X}\left\{s_{i}(x)+\frac{1}{2 \alpha_{k}}\left\|x-z_{k}\right\|^{2}\right\} \quad i=1, \ldots, m ;
\end{aligned}
$$

Algoritmo Proximal 4

$$
x_{k, i}=\arg \min _{x \in X}\left\{s_{i}(x)+\tilde{\nabla} h_{i}\left(x_{k, i-1}\right)^{T}\left(x-x_{k, i-1}\right)+\frac{1}{2 \alpha_{k}}\left\|x-x_{k, i-1}\right\|^{2}\right\} \quad i=1, \ldots, m
$$

sendo $x_{k, 0}=x_{k}, \tilde{\nabla} h_{i}(x)$ um subgradiente de $h_{i}$ em $x$.

Todos os métodos apresentados até agora não permitem o uso de conjuntos complicados para projeção, uma vez que isto traria um custo computacional indesejável durante a execução dos mesmos. Contudo, um algoritmo inspirado no método desenvolvido por Polyak [7], permite resolver 
problemas do tipo (1.1) para conjuntos que possam ser representados na forma

$$
X=\bigcap_{i=1}^{p} \operatorname{lev}_{0}\left(h_{i}\right),
$$

sendo $h_{i}: \mathbb{R}^{n} \rightarrow \mathbb{R}$ funções convexas e $\operatorname{lev}_{\alpha}(h):=\left\{x \in \mathbb{R}^{n} \mid h(x) \leq \alpha\right\}$. Ademais, exige-se que $X$ satisfaça a Condição de Slater, ou seja, existe $\tilde{x} \in \mathbb{R}^{n}$ tal que $\phi(\tilde{x})=\max \left\{h_{i}(\tilde{x})\right\}<0$. Nestas condições, o seguinte algoritmo converge para a solução desejada:

$$
x_{k+1}:=x_{k}-\alpha_{k} v_{k}
$$

sendo $v_{k} \in \partial \phi\left(x_{k}\right)$ se $\phi\left(x_{k}\right)>0$ e $v_{k} \in \partial f\left(x_{k}\right)$ se $\phi\left(x_{k}\right) \leq 0$. Observe que o método nada mais é que o algoritmo (1.2) com uma escolha adequada de subgradientes. Quando o iterando não se encontra no conjunto factível, o algoritmo "esquece" a minimização da função custo e apenas se preocupa com a factibilização das iterações. Por outro lado, quando o iterando está em $X$, o método funciona exatamente como um algoritmo de minimização irrestrito. Neste caso, a Condição de Slater serve como garantia que em um número finito de iterações o algoritmo adentrará no conjunto factível, pois do contrário, o método pode nunca aplicar um subgradiente relacionado com a função custo, o que, obviamente, não asseguraria a convergência do algoritmo.

Em contrapartida, Helou e De Pierro apresentaram em [8] uma estrutura unificada que permite o uso de métodos incrementais juntamente com métodos de factibilização para a resolução de problemas do tipo (1.1) sem a necessidade que $X$ satisfaça a Condição de Slater. O algoritmo possui duas etapas em cada iteração e pode ser descrito como:

$$
\begin{aligned}
x_{k+1 / 2} & :=\mathscr{O}_{f}\left(\lambda_{k}, x_{k}\right) \\
x_{k} & :=\mathscr{F}_{X}\left(x_{k+1 / 2}\right),
\end{aligned}
$$

sendo $\mathscr{O}_{f}: \mathbb{R}^{n} \rightarrow \mathbb{R}^{n}$ e $\mathscr{F}_{X}: \mathbb{R}^{n} \rightarrow \mathbb{R}^{n}$ operadores que devem satisfazer:

\section{Propriedade 1}

Para determinados $\alpha>0$ e $\rho_{k}>0$, o operador $\mathscr{O}_{f}$ deve satisfazer:

$$
\left\|\mathscr{O}_{f}\left(\lambda_{k}, x_{k}\right)-x\right\|^{2} \leq\left\|x_{k}-x\right\|^{2}-\alpha \lambda_{k}\left(f\left(x_{k}\right)-f(x)\right)+\lambda_{k} \rho_{k}
$$

para todo $x \in X$;

\section{Propriedade 2}

Quando $k \rightarrow \infty$, o termo $\rho_{k}$ na propriedade acima tende a zero;

\section{Propriedade 3}


Existe $\gamma>0$ tal que $\left\|x_{k}-\mathscr{O}_{f}\left(\lambda_{k}, x_{k}\right)\right\| \leq \lambda_{k} \gamma$

\section{Propriedade 4}

Para todo $\delta>0$ existe $\varepsilon>0$ tal que para todo $x_{k+1 / 2} \operatorname{com} d_{X}\left(x_{k+1 / 2}\right) \geq \delta$ e $x \in X$, tal que

$$
\left\|\mathscr{F}_{X}\left(x_{k+1 / 2}\right)-x\right\|^{2} \leq\left\|x_{k+1 / 2}-x\right\|^{2}-\varepsilon
$$

e ainda, para todo $x \in X, \mathscr{F}_{X}(x)=x$.

Com esta nova estrutura, os autores mostraram em [9] que com um operador de factibilidade adequado, é possível convergir para a solução exata de (1.1) com

$$
X=\arg \min _{x \in C} g(x)
$$

sendo $g: \mathbb{R}^{n} \rightarrow \mathbb{R}$ um função convexa e diferenciável e $C=\mathbb{R}^{n}$. Comumente, problemas do tipo (1.1) com o conjunto factível definido como em (1.11), são chamados de problemas de otimização convexa em dois níveis, uma vez que exigem a minimização de duas funções distintas.

Problemas em dois níveis também foram estudados por Solodov. Na pesquisa apresentada em [10], o algoritmo desenvolvido converge para problemas com $f$ e $g$ diferenciáveis e $C$ sendo um conjunto simples para projeção. Como inconveniente, o método necessita de uma busca linear para cada iteração. Já para o caso em que ambas as funções não são diferenciáveis, o trabalho exposto em [11] garante a convergência da solução para $C=\mathbb{R}^{n}$. Contudo, como o estudo considera funções não diferenciáveis, há a necessidade do uso da técnica conhecida como bundle [1,12], a qual permite encontrar uma direção de descida para cada iteração, mas em contrapartida, necessita de soluções de subproblemas durante a execução do algoritmo. Fundamentalmente, os métodos descritos consistem, em cada iteração, encontrar uma direção de descida para a função $F_{\sigma_{k}}(x):=$ $\sigma_{k} f(x)+g(x)$, com $\sigma_{k} \rightarrow 0^{+}$. Vale ressaltar que, atualmente, este último estudo abrange uma das maiores classes de problemas de minimização convexa passíveis de solução.

\subsection{Objetivo e Contribuições}

O intuito deste estudo é ampliar a classe de problemas de otimização convexa que podem ser resolvidos de forma exata, tendo como objetivo a solução do seguinte problema:

$$
\begin{aligned}
& \text { Min: } f(x) \\
& \text { s.a: } x \in \underset{w \in C}{\arg \min } g(w),
\end{aligned}
$$


sendo $f, g: \mathbb{R}^{n} \rightarrow \mathbb{R}$ funções convexas não necessariamente diferenciáveis e $C \subset \mathbb{R}^{n}$ um conjunto convexo representado por

$$
C=\bigcap_{i=1}^{p} \operatorname{lev}_{0}\left(h_{i}\right) .
$$

Mostraremos no decorrer deste trabalho que não é necessário o uso da projeção em $C$ para que o método convirja e, ao contrário dos métodos obtidos em [9-11], não exigimos nenhuma busca linear ou resolução de subproblemas durante a execução dos nossos algoritmos.

Finalmente, além da motivação teórica de solucionarmos problemas antes inviáveis de forma prática, temos também como aplicação para esta pesquisa a reconstrução de imagens tomográficas, uma vez que inúmeros métodos de reconstrução utilizados na tomografia tem como base a solução de um problema de otimização convexa. 


\section{Novos Métodos de Minimização em Dois Níveis}

Neste capítulo apresentamos dois novos algoritmos que têm como objetivo resolver problemas de minimização convexa em dois níveis de modo mais geral e flexível do que foi feito em [911]. Ademais, mostramos os principais resultados de convergência, bem como os operadores que satisfazem as propriedades exigidas pelos métodos.

\subsection{Resultados Preliminares, Definições e Notações}

Para que as provas dos resultados de convergência sejam facilitadas, apresentamos antes alguns resultados que aparecem com frequência nas demonstrações posteriores. Além disso, exibimos algumas notações e definições úteis.

Definição 2.1 Dado um ponto $x$ e um conjunto $X$, definimos a projeção de $x$ em $X$ como sendo $\mathscr{P}_{X}(x):=\underset{y \in X}{\arg \min }\|y-x\|$. Desta forma, a distância de um ponto $x$ a um conjunto $X$ é definida como $d_{X}(x):=\left\|x-\mathscr{P}_{X}(x)\right\|$.

Observemos que a projeção está bem definida para um conjunto $X$ convexo e fechado. Uma vez que o conjunto $X$ é fechado, segue que o conjunto de projeção para cada ponto no espaço é não-vazio. Suponha agora que o conjunto de elementos que são a projeção de um determinado $z$ arbitrário em $X$ possui dois ou mais elementos. Deste modo, $x_{1} \in \mathscr{P}_{X}(z)$ e $x_{2} \in \mathscr{P}_{X}(z)$ para certos $x_{1}, x_{2} \in X$ e $x_{1} \neq x_{2}$. Definimos agora $x:=\left(x_{1}+x_{2}\right) / 2$. Como $X$ é convexo segue que $x \in X$. Assim,

$$
\begin{aligned}
\left\|z-\left(x_{1}+x_{2}\right) / 2\right\| & =\left\|z / 2-x_{1} / 2+z / 2-x_{2} / 2\right\| \\
& \leq 1 / 2\left\|z-x_{1}\right\|+1 / 2\left\|z-x_{2}\right\| \\
& =\left\|z-x_{1}\right\| \\
& =\left\|z-x_{2}\right\| .
\end{aligned}
$$


Observe que esta desigualdade só assume a igualdade quando $z-x_{1} \mathrm{e} z-x_{2}$ são vetores linearmente dependentes, ou seja, como $\left\|z-x_{1}\right\|=\left\|z-x_{2}\right\|$, só teríamos igualdade quando $x_{1}=x_{2}$, mas como assumimos o oposto segue que $\left\|z-\left(x_{1}+x_{2}\right) / 2\right\|<\left\|z-x_{1}\right\|=\left\|z-x_{2}\right\|$. Absurdo, pois pela definição de projeção isto não pode ocorrer. Logo, quando existe projeção em um conjunto convexo, ela é única.

A seguir enunciamos duas propriedades da projeção, cujas demonstrações podem ser vistas em [1].

Teorema 2.1 Seja X um conjunto não vazio, convexo e fechado. Então $x \in X$ é a projeção de $z$ em $X$ se, e só se,

$$
\langle z-x, y-x\rangle \leq 0, \forall y \in X
$$

Proposição 2.1 Seja X um conjunto não vazio, convexo e fechado. Então

$$
\left\|\mathscr{P}_{X}\left(x_{1}\right)-\mathscr{P}_{X}\left(x_{2}\right)\right\|^{2} \leq\left\langle\mathscr{P}_{X}\left(x_{1}\right)-\mathscr{P}_{X}\left(x_{2}\right), x_{1}-x_{2}\right\rangle
$$

Com este último resultado, segue de imediato por Cauchy-Schwarz que a projeção sobre um conjunto convexo e fechado é uma função não expansiva, ou seja,

$$
\left\|\mathscr{P}_{X}\left(x_{1}\right)-\mathscr{P}_{X}\left(x_{2}\right)\right\| \leq\left\|x_{1}-x_{2}\right\|
$$

Feitas estas considerações sobre a projeção em um conjunto convexo, nos concentremos no conceito de convergência de conjuntos.

Definição 2.2 Dado um conjunto convexo e compacto $X \subset \mathbb{R}^{n}$, definimos o seguinte conjunto:

$$
X^{\varepsilon}:=\left\{x \in \mathbb{R}^{n} \mid\left\|x-\mathscr{P}_{X}(x)\right\|<\varepsilon\right\} .
$$

Definição 2.3 Seja $\left\{X_{n}\right\}$ uma sequência de conjuntos convexos e compactos com $X_{n} \subset \mathbb{R}^{n}$. Dizemos que $\left\{X_{n}\right\}$ converge para um determinado conjunto convexo e compacto $X \subset \mathbb{R}^{n}$ (notação: $\left.X_{n} \rightarrow X\right)$ se para todo $\varepsilon>0$ existe $n_{0}$ tal que para $n>n_{0}$ temos

$$
X_{n} \subset X^{\varepsilon} \quad e \quad X \subset X_{n}^{\varepsilon}
$$

Com estas definições em mãos, podemos enunciar um resultado que pode ser encontrado em [13]:

Lema 2.1 Sejam $\left\{X_{n}\right\}$ uma sequência limitada de conjuntos convexos e compactos com $X_{n} \subset \mathbb{R}^{n}$ e $X \subset \mathbb{R}^{n}$ um conjunto também convexo e compacto. Assim, $X_{n} \rightarrow X$ se, e só se, as seguintes condições acontecem: 
i) Se $x \in X$, então existem $x_{n} \in X_{n}$ tal que $x_{n} \rightarrow x$;

ii) Se $x_{n_{k}} \in X_{n_{k}}$ e $x_{n_{k}} \rightarrow x$, então $x \in X$.

Prova. Vamos supor inicialmente que $X_{n} \rightarrow X$. Seja $x \in X$ um elemento qualquer e $\varepsilon>0$ um número real arbitrário. Assim, tome $x_{n}=\mathscr{P}_{X_{n}}(x) \in X_{n}$. Logo, como $X \subset X_{n}^{\varepsilon}$ para um $n$ suficientemente grande, segue que $\left\|x-\mathscr{P}_{X_{n}}(x)\right\|<\varepsilon$, e portanto, $x_{n} \rightarrow x$. Agora, seja $x_{n_{k}} \in X_{n_{k}} \operatorname{com} x_{n_{k}} \rightarrow x$. Desta forma, para $\varepsilon>0$ existe $n_{0}$ tal que se $n>n_{0}$ temos $x_{n_{k}} \in X^{\varepsilon}$ e $\left\|x-x_{n_{k}}\right\|<\varepsilon$. Logo,

$$
\left\|x-\mathscr{P}_{X}\left(x_{n_{k}}\right)\right\| \leq\left\|x-x_{n_{k}}\right\|+\left\|x_{n_{k}}-\mathscr{P}_{X}\left(x_{n_{k}}\right)\right\|<2 \varepsilon .
$$

Portanto, $x \in X$, uma vez que $X$ é compacto.

Reciprocamente, suponha que $X_{n} \nRightarrow X$. Desta forma, temos duas opções:

a) Existe $\varepsilon>0$ e infinitos $n$ 's tal que $X \nsubseteq X_{n}^{\varepsilon}$;

b) Existe $\varepsilon>0$ e infinitos $n$ 's tal que $X_{n} \nsubseteq X^{\varepsilon}$.

Suponha que a) aconteça. Assim, existe $x \in X$ e infinitos $n$ 's tal que $\left\|x-\mathscr{P}_{X_{n}}(x)\right\| \geq \varepsilon$, contradizendo i). Ainda, se supusermos que b) é verdade, então podemos assumir uma subsequência $\left\{x_{n_{k}}\right\}$ tal que $x_{n_{k}} \rightarrow x$ (devido à limitação de $\left.\left\{X_{n}\right\}\right)$ e $x \notin X$, contradizendo ii).

Lema 2.2 Seja $\left\{X_{n}\right\}$ uma sequência de conjuntos convexos e compactos com $X_{n} \subset \mathbb{R}^{n}$. Se $X_{n} \rightarrow X$ para algum $X \subset \mathbb{R}^{n}$ convexo e compacto, então
a) $\lim _{n \rightarrow \infty}\left[\max _{x \in X_{n}} d_{X}(x)\right] \rightarrow 0$
b) $\lim _{n \rightarrow \infty}\left[\max _{x \in X} d_{X_{n}}(x)\right] \rightarrow 0$.

Prova. Mostremos o item a) primeiro. Como $X_{n} \rightarrow X$, segue por definição que dado $\varepsilon>0$ existe $n_{0}$ tal que se $n>n_{0}$ então $X_{n} \subset X^{\varepsilon}$, ou seja, $\left\|x-\mathscr{P}_{X}(x)\right\|<\varepsilon$ para todo $x \in X_{n}$. Desta forma, para $n>n_{0}$ temos

$$
\max _{x \in X_{n}} d_{X}(x)<\varepsilon .
$$

Como $\varepsilon>0$ é arbitrário o resultado segue.

Demonstremos o segundo resultado. Pela hipótese de convergência temos que dado $\varepsilon>0$ existe $n_{0}$ tal que se $n>n_{0}$ então $X \subset X_{n}^{\varepsilon}$, ou seja, $\left\|x-\mathscr{P}_{X_{n}}(x)\right\|<\varepsilon$ para todo $x \in X$. Desta forma, 
para $n>n_{0}$ temos

$$
\max _{x \in X} d_{X_{n}}(x)<\varepsilon
$$

Novamente, como $\varepsilon>0$ é arbitrário, temos o resultado.

Lema 2.3 Sejam $\left\{X_{n}\right\}$ e $\left\{Y_{n}\right\}$ duas sequências de conjuntos convexos e compactos com $X_{n}, Y_{n} \subset \mathbb{R}^{n}$. Se $X_{n} \rightarrow X$ e $Y_{n} \rightarrow X$ para algum $X \subset \mathbb{R}^{n}$ convexo e compacto, então

$$
\lim _{n \rightarrow \infty}\left[\max _{x \in X_{n}} d_{Y_{n}}(x)\right] \rightarrow 0
$$

Prova. Notemos que

$$
\begin{aligned}
\max _{x \in X_{n}} d_{Y_{n}}(x)= & \max _{x \in X_{n}}\left\|x-\mathscr{P}_{Y_{n}}(x)\right\| \\
= & \max _{x \in X_{n}}\left\|x-\mathscr{P}_{X}(x)+\mathscr{P}_{X}(x)-\mathscr{P}_{Y_{n}}(x)\right\| \\
\leq & \max _{x \in X_{n}}\left\|x-\mathscr{P}_{X}(x)\right\|+\max _{x \in X_{n}}\left\|\mathscr{P}_{X}(x)-\mathscr{P}_{Y_{n}}(x)\right\| \\
\leq & \max _{x \in X_{n}}\left\|x-\mathscr{P}_{X}(x)\right\|+\max _{x \in X_{n}}\left\|\mathscr{P}_{X}(x)-\mathscr{P}_{Y_{n}}\left(\mathscr{P}_{X}(x)\right)\right\| \\
& +\max _{x \in X_{n}}\left\|\mathscr{P}_{Y_{n}}\left(\mathscr{P}_{X}(x)\right)-\mathscr{P}_{Y_{n}}(x)\right\| \\
\leq & \max _{x \in X_{n}}\left\|x-\mathscr{P}_{X}(x)\right\|+\max _{x \in X_{n}}\left\|\mathscr{P}_{X}(x)-\mathscr{P}_{Y_{n}}\left(\mathscr{P}_{X}(x)\right)\right\| \\
& +\max _{x \in X_{n}}\left\|\mathscr{P}_{X}(x)-x\right\| \\
\leq & \max _{x \in X_{n}} 2 d_{X}(x)+\max _{x \in X} d_{Y_{n}}(x) .
\end{aligned}
$$

Logo, pelo lema anterior temos o resultado desejado.

Apresentamos ainda alguns resultados de grande valia para o entendimento das demonstrações posteriores.

Lema 2.4 Sejam $Z \subset W$ dois conjuntos fechados, convexos e não-vazios. Então

$$
d_{Z}(x) \leq d_{Z}\left(\mathscr{P}_{W}(x)\right)+2 d_{W}(x) .
$$

Prova. Usando a desigualdade triangular junto com a propriedade que garante a não-expansividade da projeção, temos

$$
\begin{aligned}
\left\|x-\mathscr{P}_{Z}(x)\right\| & \leq\left\|x-\mathscr{P}_{W}(x)\right\|+\left\|\mathscr{P}_{W}(x)-\mathscr{P}_{Z} \mathscr{P}_{W}(x)\right\|+\left\|\mathscr{P}_{Z} \mathscr{P}_{W}(x)-\mathscr{P}_{Z}(x)\right\| \\
& \leq\left\|x-\mathscr{P}_{W}(x)\right\|+\left\|\mathscr{P}_{W}(x)-\mathscr{P}_{Z} \mathscr{P}_{W}(x)\right\|+\left\|\mathscr{P}_{W}(x)-x\right\|,
\end{aligned}
$$


que prova a afirmação.

Lema 2.5 Seja $F$ uma função convexa e assuma que o conjunto $Z^{*}$ de minimizadores de $F$ sobre o conjunto $Z$ convexo e fechado é não-vazio e limitado. Então, para $\delta>0$ arbitrário, o conjunto $Z_{\delta}^{*}:=\left\{x \in Z: F(x) \leq F^{*}+\delta\right\}$, sendo $F^{*}$ o menor valor de $F$ sobre $Z$, é também limitado.

Prova. Defina $f: \mathbb{R}^{n} \rightarrow(-\infty, \infty]$ como

$$
f(x)= \begin{cases}F(x) & \text { se } \quad x \in Z \\ \infty & \text { caso contrário }\end{cases}
$$

e use [14, Proposição 2.3.1].

Além dos resultados acima, usaremos, daqui por diante, as seguintes notações:

- $f_{X}^{*}$ é o valor ótimo de $f$ sobre $X$;

- $D_{X}^{f}(\delta):=\left\{x \in\left\{\mathscr{P}_{X}\left(x_{k}\right)\right\}: f(x) \leq f_{X}^{*}+\delta\right\}$;

- $X_{f}^{*}:=\left\{x \in X: f(x) \leq f_{X}^{*}\right\}$

- $d_{X}^{f}(\delta):=\sup _{x \in D_{X}^{f}(\delta)} d_{X_{f}^{*}}(x)$;

- $X=C_{g}^{*}$.

Antes de apresentarmos os principais resultados que culminam na convergência do algoritmo, exibimos uma última proposição.

Proposição 2.2 Sejam $f: \mathbb{R}^{m} \rightarrow \mathbb{R}$ uma função convexa, $X \subset \mathbb{R}^{n}$ um conjunto convexo e fechado, $X_{f}^{*}$ limitado e $\left\{x_{k}\right\}$ uma sequência qualquer em $\mathbb{R}^{n}$. Então,

$$
\lim _{\delta \rightarrow 0} d_{X}^{f}(\boldsymbol{\delta})=0
$$

Prova. Seja $\left\{\delta_{n}\right\} \subset \mathbb{R}_{+}$uma sequência qualquer tal que $\delta_{n} \rightarrow 0$. Notemos que pelo Lema 2.5, temos que os conjuntos

$$
\bar{X}_{\delta_{n}}:=\left\{x \in X: f(x) \leq f_{X}^{*}+\delta_{n}\right\}
$$


são limitados para qualquer $\delta_{n}$. Mais ainda, a sequência $\left\{X_{\delta_{n}}\right\}$ é limitada. Além disso, os conjuntos $\bar{X}_{\delta}$ são todos convexos e fechados.

Seja agora $x \in X_{f}^{*}$ qualquer. Observe que desta forma é possível encontrar uma sequência $\left\{x_{n}\right\}$ $\operatorname{com} x_{n} \in \bar{X}_{\delta_{n}}$ tal que $x_{n} \rightarrow x$ (basta fazermos $x_{n}=x$ ). Por outro lado, se supusermos que existe $\left\{x_{n}\right\}$ $\operatorname{com} x_{n} \in \bar{X}_{\delta_{n}}$ tal que $x_{n} \rightarrow x$ para algum $x \in \mathbb{R}^{n}$, temos de imediato que $x \in X_{f}^{*}$. De fato, como $X$ é fechado e $f$ é contínua, temos que $x \in X$ e

$$
\lim _{n \rightarrow \infty} f\left(x_{n}\right) \leq \lim _{n \rightarrow \infty}\left(f_{X}^{*}+\delta_{n}\right) \Rightarrow f(x) \leq f_{X}^{*}
$$

Desta forma, pelo Lema 2.1, segue que $X_{\delta_{n}} \rightarrow X_{f}^{*}$, e portanto, pelo Lema 2.2, temos que

$$
\lim _{\delta \rightarrow 0} \sup _{x \in \bar{X}_{\delta}} d_{X_{f}^{*}}(x)=0
$$

Agora, uma vez que

$$
d_{X}^{f}(\boldsymbol{\delta}) \leq \sup _{x \in \bar{X}_{\delta}} d_{X_{f}^{*}}(x)
$$

temos de imediato o resultado desejado.

\subsection{Algoritmo I}

Dado $x_{0} \in \mathbb{R}^{n}$, definimos uma iteração $k$ do nosso algoritmo como

$$
\begin{aligned}
x_{k+1 / 3} & :=\mathscr{O}_{f}\left(\lambda_{k}, x_{k}\right) \\
x_{k+2 / 3} & :=\mathscr{O}_{g}\left(\mu_{k}, x_{k+1 / 3}\right) \\
x_{k+1} & :=\mathscr{F}_{C}\left(\mu_{k}, x_{k+2 / 3}\right),
\end{aligned}
$$

sendo que os operadores $\mathscr{O}_{f}$ e $\mathscr{O}_{g}$ precisam satisfazer as mesmas propriedades 1-3 do operador de otimalidade exigidas em [8]. Para maior clareza, apresentamos novamente as três propriedades e a nova condição do operador de factibilidade exigida pelo método.

\subsubsection{Propriedades}

Para uma função convexa $f: \mathbb{R}^{n} \rightarrow \mathbb{R}$ e passos $\lambda_{k}>0$ fixados, dizemos que $\mathscr{O}_{f}$ é um operador de otimalidade se as seguintes condições forem válidas: 
Propriedade 1 Para determinados $\beta>0$ e $\rho_{k}^{f}>0$, o operador deve satisfazer:

$$
\left\|\mathscr{O}_{f}\left(\lambda_{k}, x_{k}\right)-x\right\|^{2} \leq\left\|x_{k}-x\right\|^{2}-\beta \lambda_{k}\left(f\left(x_{k}\right)-f(x)\right)+\lambda_{k} \rho_{k}^{f}
$$

para todo $x \in C$.

Propriedade 2 Quando $k \rightarrow \infty$, o termo $\rho_{k}^{f}$ na propriedade acima tende a zero.

Propriedade 3 Existe $\gamma>0$ tal que

$$
\left\|x_{k}-\mathscr{O}_{f}\left(\lambda_{k}, x_{k}\right)\right\| \leq \lambda_{k} \gamma
$$

Além disto, dizemos que $\mathscr{F}_{C}$ é um operador de factibilidade se a seguinte propriedade é satisfeita:

Propriedade 4 Para todo $k \in \mathbb{N}$ e $x \in C$, temos que

$$
\left\|\mathscr{F}_{C}\left(\mu_{k}, x_{k+2 / 3}\right)-x\right\|^{2} \leq\left\|x_{k+2 / 3}-x\right\|^{2}
$$

e devem existir $E>0$ e $\varepsilon>0$ tal que

$$
d_{C}\left(\mathscr{F}_{C}\left(\mu_{k}, x_{k+2 / 3}\right)\right) \leq E \mu_{k}^{\varepsilon}
$$

Ademais, $\mathscr{F}_{C}\left(\mu_{k}, x_{k+2 / 3}\right)=x_{k+2 / 3}$ para todo $x_{k+2 / 3} \in C$.

É importante ressaltar que apesar da propriedade 4 exigir que (2.4) valha, mostraremos na seção 2.6 que não é necessário calcular $d_{C}\left(\mathscr{F}_{C}\left(\mu_{k}, x_{k+2 / 3}\right)\right)$ em nenhuma iteração do algoritmo se usarmos um operador de factibilidade adequado.

\subsubsection{Resultados Principais}

Lema 2.6 Suponha que $\left\{x_{k}\right\}$ é uma sequência gerada pelo Algoritmo I, $\mathscr{F}_{X}$ satisfaça (2.3), $\mathscr{O}_{f}$ satisfaça a Propriedade 3, $\mathscr{O}_{g}$ satisfaça as Propriedades 1-3, X e $d_{X}\left(x_{k}\right)$ sejam limitados, $d_{C}\left(x_{k}\right) \rightarrow$ $0, \sum_{k} \mu_{k}=\infty, \mu_{k} \rightarrow 0^{+}$e $\lambda_{k} / \mu_{k} \rightarrow 0$. Suponha ainda que exista $M$ tal que $\forall z \in\left\{x_{k}, \mathscr{P}_{C}\left(x_{k}\right)\right\}$ existe $v \in \partial g(z)$ tal que $\|v\|<M$. Então,

$$
\lim _{k \rightarrow \infty} d_{X}\left(x_{k}\right)=0
$$


Prova. Observemos inicialmente que a definição de distância seguida por (2.3) e pela Propriedade 1 de $\mathscr{O}_{g}$, nos diz que

$$
\begin{aligned}
d_{X}^{2}\left(x_{k+1}\right) & \leq\left\|x_{k+1}-\mathscr{P}_{X}\left(x_{k+1 / 3}\right)\right\|^{2}=\left\|\mathscr{F}_{C}\left(x_{k+2 / 3}\right)-\mathscr{P}_{X}\left(x_{k+1 / 3}\right)\right\|^{2} \\
& \leq\left\|x_{k+2 / 3}-\mathscr{P}_{X}\left(x_{k+1 / 3}\right)\right\|^{2} \\
& \leq\left\|x_{k+1 / 3}-\mathscr{P}_{X}\left(x_{k+1 / 3}\right)\right\|^{2}-\beta \mu_{k}\left(g\left(x_{k+1 / 3}\right)-g_{C}^{*}\right)+\mu_{k} \rho_{k}^{f} \\
& =d_{X}^{2}\left(x_{k+1 / 3}\right)-\beta \mu_{k}\left(g\left(x_{k+1 / 3}\right)-g_{C}^{*}\right)+\mu_{k} \rho_{k}^{f},
\end{aligned}
$$

Usando a Propriedade 3 de $\mathscr{O}_{f}$ temos:

$$
\begin{aligned}
d_{X}^{2}\left(x_{k+1 / 3}\right) & =\left\|x_{k+1 / 3}-\mathscr{P}_{X}\left(x_{k+1 / 3}\right)\right\|^{2} \\
& \leq\left\|x_{k+1 / 3}-\mathscr{P}_{X}\left(x_{k}\right)\right\|^{2} \\
& \leq\left(\left\|x_{k+1 / 3}-x_{k}\right\|+d_{X}\left(x_{k}\right)\right)^{2} \\
& \leq\left(\lambda_{k} \gamma+d_{X}\left(x_{k}\right)\right)^{2} \\
& \leq d_{X}^{2}\left(x_{k}\right)+\lambda_{k} \bar{M},
\end{aligned}
$$

sendo $\bar{M}=\gamma^{2} \sup \left\{\lambda_{k}\right\}+2 \gamma \sup \left\{d_{X}\left(x_{k}\right)\right\}$. Portanto, por (2.5) e (2.6), segue que

$$
d_{X}^{2}\left(x_{k+1}\right) \leq d_{X}^{2}\left(x_{k}\right)+\lambda_{k} \bar{M}+\mu_{k} \rho_{k}^{f}-\beta \mu_{k}\left(g\left(x_{k+1 / 3}\right)-g_{C}^{*}\right) .
$$

Considerando a limitação sobre $\partial g\left(x_{k}\right)$ e a Propriedade 3 de $\mathscr{O}_{f}$, temos que

$$
g\left(x_{k+1 / 3}\right)-g\left(x_{k}\right)>-M\left\|x_{k+1 / 3}-x_{k}\right\|>-\lambda_{k} M \gamma .
$$

Portanto,

$$
d_{X}^{2}\left(x_{k+1}\right)<d_{X}^{2}\left(x_{k}\right)+\lambda_{k} \bar{M}+\mu_{k} \rho_{k}^{f}+\lambda_{k} \mu_{k} M \gamma \beta-\beta \mu_{k}\left(g\left(x_{k}\right)-g_{C}^{*}\right) .
$$

Agora, seja $\delta>0$ um número real arbitrário. Lembrando que $\lambda_{k} / \mu_{k} \rightarrow 0$ e que a Propriedade 2 de $O_{g}$ é válida, segue que para um $k$ suficientemente grande as seguintes desigualdades são satisfeitas:

$$
\frac{\lambda_{k}}{\mu_{k}} \bar{M}<\beta \frac{\delta}{8}, \quad \rho_{k}^{f}<\beta \frac{\delta}{8}, \quad \lambda_{k} M \gamma \beta<\beta \frac{\delta}{8} \quad \text { e } \quad M d_{C}\left(x_{k}\right)<\frac{\delta}{2}
$$

Consideremos, neste momento, duas situações complementares:

1. $d_{X}\left(\mathscr{P}_{C}\left(x_{k}\right)\right)>d_{C}^{g}(\boldsymbol{\delta})$;

2. $d_{X}\left(\mathscr{P}_{C}\left(x_{k}\right)\right) \leq d_{C}^{g}(\delta)$. 
Vamos supor inicialmente que estamos no caso 1. Assim, pela limitação de $\partial g\left(\mathscr{P}_{C}\left(x_{k}\right)\right)$, temos que $g\left(x_{k}\right) \geq g\left(\mathscr{P}_{C}\left(x_{k}\right)\right)-M d_{C}\left(x_{k}\right)>g_{C}^{*}+\delta / 2$. Ademais, por (2.7) e (2.8), segue que

$$
\begin{aligned}
\frac{d_{X}^{2}\left(x_{k+1}\right)}{\mu_{k}} & <\frac{d_{X}^{2}\left(x_{k}\right)}{\mu_{k}}+\frac{\lambda_{k}}{\mu_{k}} \bar{M}+\rho_{k}^{f}+\lambda_{k} M \gamma \beta-\beta\left(g\left(x_{k}\right)-g^{*}\right) \\
& <\frac{d_{X}^{2}\left(x_{k}\right)}{\mu_{k}}+\beta \frac{\delta}{8}+\beta \frac{\delta}{8}+\beta \frac{\delta}{8}-\beta \frac{\delta}{2} \\
& =\frac{d_{X}^{2}\left(x_{k}\right)}{\mu_{k}}-\beta \frac{\delta}{8} .
\end{aligned}
$$

Consequentemente,

$$
d_{X}^{2}\left(x_{k+1}\right)<d_{X}^{2}\left(x_{k}\right)-\mu_{k} \beta \frac{\delta}{8} .
$$

Uma vez que $\sum_{k} \mu_{k}=\infty$, segue que o próximo caso ocorre infinitas vezes.

Vamos supor agora que o caso 2 ocorra. Assim, usando (2.3) e a Propriedade 3 dos operadores $O_{g}$ e $O_{f}$, obtemos:

$$
\begin{aligned}
d_{X}\left(x_{k+1}\right) & \leq\left\|x_{k+1}-\mathscr{P}_{X}\left(x_{k}\right)\right\|=\left\|\mathscr{F}_{C}\left(x_{k+2 / 3}\right)-\mathscr{P}_{X}\left(x_{k}\right)\right\| \\
& \leq\left\|x_{k+2 / 3}-\mathscr{P}_{X}\left(x_{k}\right)\right\| \\
& \leq\left\|x_{k+2 / 3}-x_{k+1 / 3}\right\|+\left\|x_{k+1 / 3}-\mathscr{P}_{X}\left(x_{k}\right)\right\| \\
& \leq \mu_{k} \gamma+\left\|x_{k+1 / 3}-\mathscr{P}_{X}\left(x_{k}\right)\right\| \\
& \leq \mu_{k} \gamma+\left\|x_{k+1 / 3}-x_{k}\right\|+\left\|x_{k}-\mathscr{P}_{X}\left(x_{k}\right)\right\| \\
& \leq d_{X}\left(x_{k}\right)+\left(\mu_{k}+\lambda_{k}\right) \gamma .
\end{aligned}
$$

Portanto, usando este último resultado com o Lema 2.4 segue que

$$
\begin{aligned}
d_{X}\left(x_{k+1}\right) & \leq d_{X}\left(\mathscr{P}_{C}\left(x_{k}\right)\right)+2 d_{C}\left(x_{k}\right)+\left(\mu_{k}+\lambda_{k}\right) \gamma \\
& \leq d_{C}^{g}(\delta)+2 d_{C}\left(x_{k}\right)+\left(\mu_{k}+\lambda_{k}\right) \gamma
\end{aligned}
$$

Desta forma, como $\lambda_{k} \rightarrow 0, \mu_{k} \rightarrow 0$ e $d_{C}\left(x_{k}\right) \rightarrow 0$, segue que

$$
\limsup _{k \rightarrow \infty} d_{X}\left(x_{k}\right) \leq d_{C}^{g}(\boldsymbol{\delta}),
$$

e portanto a afirmação é verdadeira já que a desigualdade é válida para todo $\delta>0$ e pela Proposição 2.2 sabemos que $\lim _{\delta \rightarrow 0} d_{C}^{g}(\boldsymbol{\delta})=0$.

Notemos que a hipótese de que $\lambda_{k} / \mu_{k} \rightarrow 0$ é de suma importância para que o conjunto factível seja respeitado, uma vez que esta propriedade garante que o operador $\mathscr{O}_{g}$ seja mais "forte" "assintoticamente"do que o operador $\mathscr{O}_{f}$. Finalmente, apresentamos o principal resultado de convergência. 
Lema 2.7 Suponha que $\left\{x_{k}\right\}$ é uma sequência gerada pelo Algoritmo I, $\mathscr{F}_{C}$ satisfaz a Propriedade 4 com $\varepsilon$ tal que $\mu_{k}^{1+\varepsilon} / \lambda_{k} \rightarrow 0$ e $\mathscr{O}_{f}$ e $\mathscr{O}_{g}$ obedecem as Propriedades 1-3 com $\mathscr{O}_{g}$ satisfazendo também $\mu_{k} \rho_{k}^{g} / \lambda_{k} \rightarrow 0$. Ademais, assuma que $X^{*} e\left\{d_{X^{*}}\left(x_{k}\right)\right\}$ são limitados, $d_{X}\left(x_{k}\right) \rightarrow 0$, $\sum_{k} \lambda_{k}=\infty, \lambda_{k} \rightarrow 0^{+}$, e existe $M$ tal que $\forall z \in\left\{x_{k}, \mathscr{P}_{C}\left(x_{k}\right)\right\}$, existe $v \in \partial g(z)$ com $\|v\|<M$ e para todo $z \in\left\{\mathscr{P}_{X}\left(x_{k}\right)\right\}$, existe $v \in \partial f(z)$ com $\|v\|<M$. Então

$$
\lim _{k \rightarrow \infty} d_{X^{*}}\left(x_{k}\right)=0
$$

Prova. Primeiramente, notemos que usando (2.3) e a Propriedade 1 de $\mathscr{O}_{g}$ e $\mathscr{O}_{f}$, temos:

$$
\begin{aligned}
d_{X^{*}}^{2}\left(x_{k+1}\right) & \leq\left\|x_{k+1}-\mathscr{P}_{X^{*}}\left(x_{k}\right)\right\|^{2}=\left\|\mathscr{F}_{C}\left(x_{k+2 / 3}\right)-\mathscr{P}_{X^{*}}\left(x_{k}\right)\right\|^{2} \\
& \leq\left\|x_{k+2 / 3}-\mathscr{P}_{X^{*}}\left(x_{k}\right)\right\|^{2} \\
& \leq\left\|x_{k+1 / 3}-\mathscr{P}_{X^{*}}\left(x_{k}\right)\right\|^{2}+\mu_{k} \rho_{k}^{g}-\beta \mu_{k}\left(g\left(x_{k+1 / 3}\right)-g_{C}^{*}\right) \\
& \leq d_{X^{*}}^{2}\left(x_{k}\right)+\lambda_{k} \rho_{k}^{f}+\mu_{k} \rho_{k}^{g}-\beta \lambda_{k}\left(f\left(x_{k}\right)-f_{X}^{*}\right)-\beta \mu_{k}\left(g\left(x_{k+1 / 3}\right)-g_{C}^{*}\right),
\end{aligned}
$$

Pela limitação assumida de $\partial g\left(\mathscr{P}_{C}\left(x_{k}\right)\right)$ e $\partial g\left(x_{k}\right)$ temos que $g\left(x_{k}\right)-g_{C}^{*} \geq g\left(x_{k}\right)-g\left(\mathscr{P}_{C}\left(x_{k}\right)\right)>$ $-M d_{C}\left(x_{k}\right)$ e $g\left(x_{k+1 / 3}\right)-g\left(x_{k}\right)>-M\left\|x_{k+1 / 3}-x_{k}\right\|$. Portanto, usando a Propriedade 3 de $\mathscr{O}_{f}$ e (2.4), segue que

$$
d_{X^{*}}^{2}\left(x_{k+1}\right) \leq d_{X^{*}}^{2}\left(x_{k}\right)+\lambda_{k} \rho_{k}^{f}+\mu_{k} \rho_{k}^{g}+\lambda_{k} \mu_{k} M \beta \gamma+\mu_{k}^{1+\varepsilon} M E \beta-\beta \lambda_{k}\left(f\left(x_{k}\right)-f_{X}^{*}\right)
$$

Agora, escolhendo $\delta>0$ um número real arbitrário e escolhendo $k_{0}$ tal que para $k \geq k_{0}$

$$
\rho_{k}^{f}<\beta \frac{\delta}{10}, \quad \frac{\mu_{k} \rho_{k}^{g}}{\lambda_{k}}<\beta \frac{\delta}{10}, \quad \mu_{k} M \beta \gamma<\beta \frac{\delta}{10}, \quad \frac{\mu_{k}^{1+\varepsilon}}{\lambda_{k}} M E \beta<\beta \frac{\delta}{10} \quad \text { e } \quad M d_{X}\left(x_{k}\right)<\frac{\delta}{2} .
$$

Consideremos, neste momento, dois casos:

1. $d_{X^{*}}\left(\mathscr{P}_{X}\left(x_{k}\right)\right)>d_{X}^{f}(\boldsymbol{\delta})$;

2. $d_{X^{*}}\left(\mathscr{P}_{X}\left(x_{k}\right)\right) \leq d_{X}^{f}(\delta)$.

Suponha que o caso 1 seja válido. Desta forma, pela limitação de $\partial f\left(\mathscr{P}_{X}\left(x_{k}\right)\right)$, obtemos que $f\left(x_{k}\right) \geq f\left(\mathscr{P}_{X}\left(x_{k}\right)\right)-M d_{X}\left(x_{k}\right)>f_{X}^{*}+\delta / 2$. Então, temos

$$
\begin{aligned}
\frac{d_{X^{*}}^{2}\left(x_{k+1}\right)}{\lambda_{k}} & \leq \frac{d_{X^{*}}^{2}\left(x_{k}\right)}{\lambda_{k}}+\rho_{k}^{f}+\frac{\mu_{k} \rho_{k}^{g}}{\lambda_{k}}+\mu_{k} M \beta \gamma+\frac{\mu_{k}^{1+\varepsilon}}{\lambda_{k}} M E \beta-\beta \delta \\
& <\frac{d_{X^{*}}^{2}\left(x_{k}\right)}{\lambda_{k}}+\beta \frac{\delta}{10}+\beta \frac{\delta}{10}+\beta \frac{\delta}{10}+\beta \frac{\delta}{10}-\beta \frac{\delta}{2} \\
& =\frac{d_{X^{*}}^{2}\left(x_{k}\right)}{\lambda_{k}}-\beta \frac{\delta}{10} .
\end{aligned}
$$


Assim,

$$
d_{X^{*}}^{2}\left(x_{k+1}\right)<d_{X^{*}}^{2}\left(x_{k}\right)-\lambda_{k} \beta \frac{\delta}{10} .
$$

Uma vez que $\sum_{k} \lambda_{k}=\infty$, o caso 2 ocorre infinitas vezes.

Vamos supor portanto que o caso 2 seja válido. Simplesmente substituindo $X$ por $X^{*}$ nos passos usados para obter (2.9) nós temos:

$$
d_{X^{*}}\left(x_{k+1}\right) \leq d_{X^{*}}\left(x_{k}\right)+\left(\mu_{k}+\lambda_{k}\right) \gamma
$$

Portanto, usando novamente o Lema 2.4, segue:

$$
\begin{aligned}
d_{X^{*}}\left(x_{k+1}\right) & \leq d_{X^{*}}\left(\mathscr{P}_{X}\left(x_{k}\right)\right)+2 d_{X}\left(x_{k}\right)+\left(\mu_{k}+\lambda_{k}\right) \gamma \\
& \leq d_{X}^{f}(\delta)+2 d_{X}\left(x_{k}\right)+\left(\mu_{k}+\lambda_{k}\right) \gamma .
\end{aligned}
$$

Uma vez que $\lambda_{k} \rightarrow 0, \mu_{k} \rightarrow 0$ e $d_{X}\left(x_{k}\right) \rightarrow 0$, segue que

$$
\limsup _{k \rightarrow \infty} d_{X^{*}}\left(x_{k}\right) \leq d_{X}^{f}(\boldsymbol{\delta})
$$

Portanto, isto mostra a afirmação, já que a inequação acima é válida para qualquer $\delta>0$ e pela Proposição 2.2 sabemos que

$$
\lim _{\delta \rightarrow 0} d_{X}^{f}(\boldsymbol{\delta})=0
$$

Desta forma, a convergência do Algoritmo I fica assegurada pelo resultado anterior. Talvez o leitor se sinta incomodado com certas hipóteses, já que elas podem parecer "fortes"demais, entretanto, mostramos nos resultados que se seguem que as hipóteses são coerentes.

Proposição 2.3 Vamos supor que $\left\{x_{k}\right\}$ é uma sequência gerada pelo Algoritmo I, $\mathscr{F}_{C}$ satisfaz (2.3), $\mathscr{O}_{f}$ e $\mathscr{O}_{g}$ satisfazem as Propriedades $1-3, X$ é limitado, $d_{C}\left(x_{k}\right) \rightarrow 0, \mu_{k} \rightarrow 0, \lambda_{k} / \mu_{k} \rightarrow 0$ e $f\left(x_{k}\right) \geq \bar{f}$ para algum $\bar{f}$. Ademais, vamos assumir que existe uma constante positiva $M$ tal que para todo $z \in\left\{x_{k}, \mathscr{P}_{C}\left(x_{k}\right)\right\}$ exista $v \in \partial g(z)$ com $\|v\|<M$. Então $\left\{d_{X}\left(x_{k}\right)\right\}$ é limitado.

Prova. Novamente, fixemos $\delta>0$ e consideramos dois casos:

1. $d_{X}\left(\mathscr{P}_{C}\left(x_{k}\right)\right)>d_{C}^{g}(\delta)$;

2. $d_{X}\left(\mathscr{P}_{C}\left(x_{k}\right)\right) \leq d_{C}^{g}(\boldsymbol{\delta})$.

Como foi feito em (2.10), usando (2.3) e pela Propriedade 1 de $\mathscr{O}_{f}$ e $\mathscr{O}_{g}$ temos, para $x \in C$, que

$$
\left\|x_{k+1}-x\right\|^{2} \leq\left\|x_{k}-x\right\|^{2}+\lambda_{k} \rho_{k}^{f}+\mu_{k} \rho_{k}^{g}-\beta \lambda_{k}\left(f\left(x_{k}\right)-f(x)\right)-\beta \mu_{k}\left(g\left(x_{k+1 / 3}\right)-g(x)\right) .
$$


Assim, usando a limitação de $\partial g\left(x_{k}\right)$ e pela Propriedade 3 de $\mathscr{O}_{f}$, temos $g\left(x_{k+1 / 3}\right)-g\left(x_{k}\right)>$ $-M \lambda_{k} \gamma$. Então, tendo $f\left(x_{k}\right) \geq \bar{f}$ em mente, denotando $\underline{f}:=\sup _{x \in X} f(x)$ e fazendo $x=\mathscr{P}_{X}\left(x_{k}\right)$, a inequação acima nos leva a:

$$
d_{X}^{2}\left(x_{k+1}\right) \leq d_{X}^{2}\left(x_{k}\right)+\lambda_{k} \rho_{k}^{f}+\mu_{k} \rho_{k}^{g}+\beta \lambda_{k}(\bar{f}-\underline{f})+\lambda_{k} \mu_{k} M \beta \gamma-\beta \mu_{k}\left(g\left(x_{k}\right)-g(x)\right) .
$$

Seja $k_{0}$ suficientemente grande tal que para todo $k>k_{0}$ temos

$$
\frac{\lambda_{k} \rho_{k}^{f}}{\mu_{k}}<\beta \frac{\delta}{10}, \quad \rho_{k}^{g}<\beta \frac{\delta}{10}, \quad \frac{\lambda_{k}}{\mu_{k}} \beta(\bar{f}-\underline{f})<\beta \frac{\delta}{10}, \quad \lambda_{k} M \beta \gamma<\beta \frac{\delta}{10} \quad \text { e } \quad M d_{C}\left(x_{k}\right)<\frac{\delta}{2} .
$$

Pressupondo que o caso 1 valha e usando a limitação de $\partial g\left(\mathscr{P}_{C}\left(x_{k}\right)\right)$, temos que $g\left(x_{k}\right)>$ $g\left(\mathscr{P}_{C}\left(x_{k}\right)\right)-M d_{C}\left(x_{k}\right)>g_{C}^{*}+\delta / 2$. Portanto, para $k>k_{0}$ :

$$
d_{X}^{2}\left(x_{k+1}\right)<d_{X}^{2}\left(x_{k}\right)-\mu_{k} \frac{\delta}{10}
$$

Já se o caso 2 é válido, então por (2.3), pela Propriedade 3 de $\mathscr{O}_{f}$ e $\mathscr{O}_{g}$ e pelo Lema 2.4, obtemos:

$$
\begin{aligned}
d_{X}\left(x_{k+1}\right) & \leq d_{X}\left(x_{k}\right)+\left(\lambda_{k}+\mu_{k}\right) \gamma \\
& \leq d_{X}\left(\mathscr{P}_{C}\left(x_{k}\right)\right)+2 d_{C}\left(x_{k}\right)+\left(\lambda_{k}+\mu_{k}\right) \gamma \\
& \leq d_{C}^{g}(\delta)+2 d_{C}\left(x_{k}\right)+\left(\lambda_{k}+\mu_{k}\right) \gamma .
\end{aligned}
$$

Uma vez que as quantidades do lado direito da inequação acima são limitadas, segue o resultado.

Assim, as hipóteses feitas tornam-se razoáveis. A única suposição que ainda pode ser restritiva é a limitação tanto de $X$ quanto de $X^{*}$. Primeiro, vale ressaltar que, para a grande maioria dos problemas de minimização convexa reais, podemos considerar um subconjunto limitado destes conjuntos, já que muitas vezes soluções com coordenadas demasiadamente grandes não possuem sentido. Ademais, uma vez que a limitação destes conjuntos só se faz necessária para garantir que tanto $d_{C}^{g}(\delta)$ quanto $d_{X}^{f}(\delta)$ convergem para zero quando $\delta \rightarrow 0$, podemos supor ao invés disso a limitação das iterações do nosso algoritmo. Desta forma, temos o seguinte resultado:

Teorema 2.2 Vamos supor que a sequência $\left\{x_{k}\right\}$ gerada pelo Algoritmo I é limitada, $\mathscr{O}_{f}$ e $\mathscr{O}_{g}$ satisfazem as Propriedades 1-3 com $\left\{\rho_{k}^{g}\right\}$ tal que $\mu_{k} \rho_{k}^{g} / \lambda_{k} \rightarrow 0$ e $\mathscr{F}_{C}$ obedece a Propriedade 4 com $\varepsilon$ tal que $\mu_{k}^{1+\varepsilon} / \lambda_{k} \rightarrow 0$. Ainda, suponha que os tamanhos de passos satisfaçam: $\lambda_{k}, \mu_{k} \rightarrow 0^{+}$, $\sum \lambda_{k}=\infty, \sum \mu_{k}=\infty$ e $\lambda_{k} / \mu_{k} \rightarrow 0$. Então

$$
d_{X^{*}}\left(x_{k}\right) \rightarrow 0 .
$$


Prova. Primeiro, a limitação de $\left\{x_{k}\right\}$ implica claramente na limitação de $d_{X}\left(x_{k}\right)$. Além disto, $\mu_{k} \rightarrow 0$ com (2.4) implica que $d_{C}\left(x_{k}\right) \rightarrow 0$. Ademais, a limitação em $\partial g$ necessária para o Lema 2.6 é satisfeita uma vez que $\left\{x_{k}\right\}$ é limitado.

Novamente pela limitação de $\left\{x_{k}\right\}$, temos que $\lim _{\delta \rightarrow 0} d_{C}^{g}(\delta)=\lim _{\delta \rightarrow 0} d_{X}^{f}(\delta)=0$, e portanto as provas dos Lemas 2.6 e 2.7 seguem da mesma forma.

\subsection{Algoritmo II}

Nesta seção apresentamos um algoritmo alternativo baseado na ideia de Polyak apresentada em (1.10), tendo a necessidade que o conjunto $C$ do problema (1.12) satisfaça a Condição de Slater. Desta forma, definimos a função $\phi: \mathbb{R}^{n} \rightarrow \mathbb{R}$ como sendo

$$
\phi(x)=\max _{1 \leq i \leq p} h_{i}(x)
$$

Assim, dado $x_{0} \in \mathbb{R}^{n}$, uma iteração $k$ do novo algoritmo é definida como:

$$
\begin{array}{r}
x_{k+1 / 2}:=\mathscr{O}_{f}\left(\lambda_{k}, x_{k}\right) ; \\
x_{k+1}:=\mathscr{F}_{X}\left(\mu_{k}, x_{k+1 / 2}\right),
\end{array}
$$

sendo

$$
\mathscr{F}_{X}\left(\mu_{k}, x_{k+1 / 2}\right)=\left\{\begin{array}{cc}
\mathscr{O}_{\phi}\left(\mu_{k}, x_{k+1 / 2}\right) & \text { se } x_{k+1 / 2} \notin C ; \\
\mathscr{O}_{g}\left(\mu_{k}, x_{k+1 / 2}\right) & \text { caso contrário. }
\end{array}\right.
$$

Todos os operadores de otimalidade devem satisfazer as mesmas Propriedades 1-3 requeridas no algoritmo anterior.

Antes de exibirmos os resultados que comprovam a convergência do algoritmo, apresentamos uma proposição útil que será usada posteriormente.

Proposição 2.4 Sejam $C$ um conjunto convexo e fechado, $g: \mathbb{R}^{n} \rightarrow \mathbb{R}$ uma função convexa, X o conjunto de pontos ótimos de g sobre $C$ e $\tilde{x} \in C$. Então, se $X$ é limitado, os seguintes conjuntos

$$
\begin{aligned}
X_{n} & :=\left(1+\frac{1}{n}\right) X+\frac{1}{n} \tilde{x} \\
X_{g_{n}} & :=\left\{x \in C \mid g(x) \leq g_{C}^{*}+\frac{1}{n}\left[g(\tilde{x})-g_{C}^{*}+1\right]\right\}
\end{aligned}
$$

convergem para $X$.

Prova. Primeiramente provemos que $X_{n} \rightarrow X$. Observe que a sequência $\left\{X_{n}\right\}$ é limitada e $X_{n}$ é 
convexo e compacto para todo $n \in \mathbb{N}$. Ademais, note que se $\bar{x} \in X$, então a sequência formada pelos elementos

$$
x_{n}=\left[\left(1+\frac{1}{n}\right) \bar{x}+\frac{1}{n} \tilde{x}\right] \in X_{n}
$$

converge para $\bar{x}$. Por outro lado, suponha que $\left\{x_{n}\right\}$ é uma sequência tal que $x_{n} \in X_{n}$ e $x_{n} \rightarrow x$ para algum $x \in \mathbb{R}^{n}$. Desta forma, como $C$ é convexo e fechado, segue que $x \in C$, e ainda, como $g$ é convexa (e portanto contínua), segue que

$$
g\left(x_{n}\right) \leq\left(1+\frac{1}{n}\right) g_{C}^{*}+\frac{1}{n} g(\tilde{x}) \Rightarrow g(x) \leq g_{C}^{*}
$$

ou seja, $x \in X$. Assim, pelo Lema 2.1, fica provada a convergência de $X_{n}$ para $X$.

Demonstremos agora que $X_{g_{n}} \rightarrow X$. Claramente, $X_{g_{n}} \subset X_{g_{1}}$, e portanto, como $X_{g_{1}}$ é limitado (pelo Lema 2.4), segue que $\left\{X_{g_{n}}\right\}$ é uma sequência limitada. Além disso, $X_{g_{n}}$ é convexo e compacto para todo $n \in \mathbb{N}$. Agora, se $\bar{x} \in X$, então a sequência constante formada pelos elementos $x_{n}=x \in X_{g_{n}}$ converge para $x$. Ademais, se supormos que $\left\{x_{n}\right\}$ é uma sequência tal que $x_{n} \in X_{n}$ e $x_{n} \rightarrow x$ para algum $x \in \mathbb{R}^{n}$, temos de imediato da continuidade de $g$ que $x \in X$. Portanto, novamente pelo Lema 2.1, segue que $X_{g_{n}} \rightarrow X$.

Finalmente, apresentamos os principais resultados de convergência que asseguram a convergência do algoritmo em questão.

Lema 2.8 Vamos assumir que $\left\{x_{k}\right\}$ é uma sequência gerada pelo Algoritmo II, o operador $\mathscr{O}_{\phi}$ satisfaz as Propriedades 1-2, $\mathscr{O}_{g}$ satisfaz as Propriedades $1-3$ e $\mathscr{O}_{f}$ satisfaz a Propriedade 3. Ademais, suponha que X é um conjunto convexo e compacto, C satisfaz a Condição de Slater, $\left\{d_{X}\left(x_{k}\right)\right\}$ é limitado, $\sum_{k} \mu_{k}=\infty, \mu_{k} \rightarrow 0^{+}$e $\lambda_{k} / \mu_{k} \rightarrow 0^{+}$. Então

$$
\lim _{n \rightarrow \infty} d_{X}\left(x_{k}\right)=0
$$

Prova. Sejam $n \in \mathbb{N}$ um valor arbitrário e $\tilde{x} \in C$ tal que $\phi(\tilde{x})<-L$, para algum $L>0 \in \mathbb{R}$ (hipótese da Condição de Slater). Desta forma, definimos

$$
X_{n}:=\left(1-\frac{1}{n}\right) X+\frac{1}{n} \tilde{x}
$$

Observe que para qualquer elemento $x_{n} \in X_{n}$ temos que

$$
\phi\left(x_{n}\right) \leq\left(1-\frac{1}{n}\right) \phi(x)+\frac{1}{n} \phi(\tilde{x}) \leq-L \frac{1}{n} .
$$


Agora, escolhemos $k_{0}$ tal que para todo $k \geq k_{0}$ segue que

$$
\frac{\lambda_{k+1}}{\mu_{k}} M<\beta \frac{z}{3}, \quad \rho_{k}^{\phi}<\beta \frac{z}{3}, \quad \rho_{k}^{g}<\beta \frac{z}{3} \quad \text { e } \quad \gamma\left(\lambda_{k+1}+\mu_{k}\right)<\frac{1}{n},
$$

sendo $z:=\min \left\{L \frac{1}{n} ; \frac{1}{n}\right\}$ e $M:=2 \gamma \sup \left\{d_{X_{n}}\left(x_{k}\right)\right\}+\gamma^{2} \sup \left\{\lambda_{k}\right\}$. Portanto, se $k \geq k_{0}$, podemos considerar 3 casos:

1. $x_{k+1 / 2} \notin C$;

2. $x_{k+1 / 2} \in C$ e $g\left(x_{k+1 / 2}\right) \geq g\left(\mathscr{P}_{X_{n}}\left(x_{k+1 / 2}\right)\right)+\frac{1}{n}$;

3. $x_{k+1 / 2} \in C$ e $g\left(x_{k+1 / 2}\right)<g\left(\mathscr{P}_{X_{n}}\left(x_{k+1 / 2}\right)\right)+\frac{1}{n}$.

Suponha que o caso 1 seja verdadeiro, então tendo em mente a Propriedade 3 do operador $\mathscr{O}_{f}$, a Propriedade 1 do operador $\mathscr{O}_{\phi}$ e (2.12), temos que

$$
\begin{aligned}
d_{X_{n}}^{2}\left(x_{k+3 / 2}\right) & =\left\|x_{k+3 / 2}-\mathscr{P}_{X_{n}}\left(x_{k+3 / 2}\right)\right\|^{2} \\
& \leq\left\|x_{k+3 / 2}-\mathscr{P}_{X_{n}}\left(x_{k+1}\right)\right\|^{2} \\
& \leq\left(\left\|x_{k+3 / 2}-x_{k+1}\right\|+\left\|x_{k+1}-\mathscr{P}_{X_{n}}\left(x_{k+1}\right)\right\|\right)^{2} \\
& \leq \lambda_{k+1} M+\left\|x_{k+1}-\mathscr{P}_{X_{n}}\left(x_{k+1}\right)\right\|^{2} \\
& \leq \lambda_{k+1} M+\left\|x_{k+1}-\mathscr{P}_{X_{n}}\left(x_{k+1 / 2}\right)\right\|^{2} \\
& \leq \lambda_{k+1} M+d_{X_{n}}^{2}\left(x_{k+1 / 2}\right)-\beta \mu_{k}\left[\phi\left(x_{k+1 / 2}\right)-\phi\left(\mathscr{P}_{X_{n}}\left(x_{k+1 / 2}\right)\right)\right]+\mu_{k} \rho_{k}^{\phi} \\
& \leq \lambda_{k+1} M+d_{X_{n}}^{2}\left(x_{k+1 / 2}\right)-\beta \mu_{k} L \frac{1}{n}+\mu_{k} \rho_{k}^{\phi} \\
& \leq \lambda_{k+1} M+d_{X_{n}}^{2}\left(x_{k+1 / 2}\right)-\beta \mu_{k} z+\mu_{k} \rho_{k}^{\phi}
\end{aligned}
$$

Assim, por (2.13), segue que

$$
\begin{aligned}
\frac{d_{X_{n}}^{2}\left(x_{k+3 / 2}\right)}{\mu_{k}} & \leq \frac{d_{X_{n}}^{2}\left(x_{k+1 / 2}\right)}{\mu_{k}}+\frac{\lambda_{k+1}}{\mu_{k}} M-\beta z+\rho_{k}^{\phi} \\
& <\frac{d_{X_{n}}^{2}\left(x_{k+1 / 2}\right)}{\mu_{k}}-\beta \frac{z}{3} .
\end{aligned}
$$

Logo,

$$
d_{X_{n}}^{2}\left(x_{k+3 / 2}\right)<d_{X_{n}}^{2}\left(x_{k+1 / 2}\right)-\beta \mu_{k} \frac{z}{3}
$$

Suponha agora que estamos no caso 2. Portanto, pela Propriedade 3 do operador $\mathscr{O}_{f}$ e pela 
Propriedade 1 do operador $\mathscr{O}_{g}$, segue que

$$
\begin{aligned}
d_{X_{n}}^{2}\left(x_{k+3 / 2}\right) & =\left\|x_{k+3 / 2}-\mathscr{P}_{X_{n}}\left(x_{k+3 / 2}\right)\right\|^{2} \\
& \leq\left\|x_{k+3 / 2}-\mathscr{P}_{X_{n}}\left(x_{k+1}\right)\right\|^{2} \\
& \leq\left(\left\|x_{k+3 / 2}-x_{k+1}\right\|+\left\|x_{k+1}-\mathscr{P}_{X_{n}}\left(x_{k+1}\right)\right\|\right)^{2} \\
& \leq \lambda_{k+1} M+\left\|x_{k+1}-\mathscr{P}_{X_{n}}\left(x_{k+1}\right)\right\|^{2} \\
& \leq \lambda_{k+1} M+\left\|x_{k+1}-\mathscr{P}_{X_{n}}\left(x_{k+1 / 2}\right)\right\|^{2} \\
& \leq \lambda_{k+1} M+d_{X_{n}}^{2}\left(x_{k+1 / 2}\right)-\beta \mu_{k}\left[g\left(x_{k+1 / 2}\right)-g\left(\mathscr{P}_{X_{n}}\left(x_{k+1 / 2}\right)\right)\right]+\mu_{k} \rho_{k}^{g} \\
& \leq \lambda_{k+1} M+d_{X_{n}}^{2}\left(x_{k+1 / 2}\right)-\beta \mu_{k} \frac{1}{n}+\mu_{k} \rho_{k}^{g} \\
& \leq \lambda_{k+1} M+d_{X_{n}}^{2}\left(x_{k+1 / 2}\right)-\beta \mu_{k} z+\mu_{k} \rho_{k}^{g} .
\end{aligned}
$$

Assim, do mesmo modo que obtemos (2.14) para o caso 1, obtemos

$$
d_{X_{n}}^{2}\left(x_{k+3 / 2}\right)<d_{X_{n}}^{2}\left(x_{k+1 / 2}\right)-\beta \mu_{k} \frac{z}{3} .
$$

Agora, suponha que estamos no caso 3. Desta forma, definimos

$$
X_{g_{n}}:=\left\{x \in C \mid g(x) \leq g_{C}^{*}+\frac{1}{n}\left[g(\tilde{x})-g_{C}^{*}+1\right]\right\} \quad \text { e } \quad d(n):=\max _{x \in X_{g_{n}}} d_{X_{n}}(x) .
$$

Consequentemente, $x_{k+1 / 2} \in X_{g_{n}}$, e portanto, pela Propriedade 3 dos operadores $\mathscr{O}_{f}$ e $\mathscr{O}_{g}$ e por (2.13), segue que

$$
\begin{aligned}
d_{X_{n}}\left(x_{k+3 / 2}\right) & \leq\left\|x_{k+3 / 2}-\mathscr{P}_{X_{n}}\left(x_{k+1 / 2}\right)\right\| \\
& \leq\left\|x_{k+3 / 2}-x_{k+1}\right\|+\left\|x_{k+1}-x_{k+1 / 2}\right\|+\left\|x_{k+1 / 2}-\mathscr{P}_{X_{n}}\left(x_{k+1 / 2}\right)\right\| \\
& \leq \gamma\left(\lambda_{k+1}+\mu_{k}\right)+d_{X_{n}}\left(x_{k+1 / 2}\right) \\
& <\frac{1}{n}+d(n) .
\end{aligned}
$$

Consequentemente, pelas desigualdades (2.14), (2.15) e (2.16), juntamente com $\sum_{k} \mu_{k}=\infty$, temos que para um $\bar{k}$ suficientemente grande a seguinte desigualdade é válida para todo $k \geq \bar{k}$ :

$$
d_{X_{n}}\left(x_{k+1 / 2}\right)<\frac{1}{n}+d(n)
$$

Notemos agora que pela Proposição 2.4, temos que $X_{n} \rightarrow X$ e $X_{g_{n}} \rightarrow X$. Desta forma, pelo Lema 2.3, segue que

$$
\lim _{n \rightarrow \infty} d(n)=0
$$


e assim, pela Propriedade 3 do operador $\mathscr{O}_{f}$ e por (2.17), segue que

$$
\lim _{k \rightarrow \infty} d_{X}\left(x_{k}\right)=0
$$

Lema 2.9 Suponha que $\left\{x_{k}\right\}$ seja uma sequência gerada pelo Algoritmo II, o operador $\mathscr{O}_{\phi}$ satisfaz as Propriedades 1-2 e $\mathscr{O}_{g}$ e $\mathscr{O}_{f}$ satisfazem as Propriedades 1-3. Ademais, suponha que X é um conjunto limitado, $d_{X}\left(x_{k}\right) \rightarrow 0, \mu_{k} \rho_{k}^{g} / \lambda_{k} \rightarrow 0, \mu_{k} \rho_{k}^{\phi} / \lambda_{k} \rightarrow 0, \sum_{k} \lambda_{k}=\infty, \mu_{k} \rightarrow 0^{+}$e $\lambda_{k} / \mu_{k} \rightarrow 0^{+}$. Por último, suponha que para todo $z \in\left\{\mathscr{P}_{X}\left(x_{k}\right)\right\}$ existe $v \in \partial f(z)$ com $\|v\| \leq W$, para algum $W \in \mathbb{R}$ fixado. Então

$$
\lim _{n \rightarrow \infty} d_{X^{*}}\left(x_{k}\right)=0
$$

Prova. Primeiramente, notemos que pela Propriedade 1 dos operadores $\mathscr{O}_{f}, \mathscr{O}_{g}$ e $\mathscr{O}_{\phi}$, obtemos:

1. Se $x_{k+1 / 2} \notin C$, então

$$
\begin{aligned}
d_{X^{*}}^{2}\left(x_{k+1}\right) & \leq\left\|x_{k+1}-\mathscr{P}_{X^{*}}\left(x_{k+1 / 2}\right)\right\|^{2} \\
& \leq\left\|x_{k+1 / 2}-\mathscr{P}_{X^{*}}\left(x_{k+1 / 2}\right)\right\|^{2}-\beta \mu_{k}\left[\phi\left(x_{k+1 / 2}\right)-\phi\left(\mathscr{P}_{X^{*}}\left(x_{k+1 / 2}\right)\right)\right]+\mu_{k} \rho_{k}^{\phi} \\
& \leq\left\|x_{k+1 / 2}-\mathscr{P}_{X^{*}}\left(x_{k}\right)\right\|^{2}+\mu_{k} \rho_{k}^{\phi} \\
& \leq d_{X^{*}}^{2}\left(x_{k}\right)-\beta \lambda_{k}\left[f\left(x_{k}\right)-f^{*}\right]+\lambda_{k} \rho_{k}^{f}+\mu_{k} \rho_{k}^{\phi}
\end{aligned}
$$

2. Se $x_{k+1 / 2} \in C$, então

$$
\begin{aligned}
d_{X^{*}}^{2}\left(x_{k+1}\right) & \leq\left\|x_{k+1}-\mathscr{P}_{X^{*}}\left(x_{k+1 / 2}\right)\right\|^{2} \\
& \leq\left\|x_{k+1 / 2}-\mathscr{P}_{X^{*}}\left(x_{k+1 / 2}\right)\right\|^{2}-\beta \mu_{k}\left[g\left(x_{k+1 / 2}\right)-g\left(\mathscr{P}_{X^{*}}\left(x_{k+1 / 2}\right)\right)\right]+\mu_{k} \rho_{k}^{g} \\
& \leq\left\|x_{k+1 / 2}-\mathscr{P}_{X^{*}}\left(x_{k}\right)\right\|^{2}+\mu_{k} \rho_{k}^{g} \\
& \leq d_{X^{*}}^{2}\left(x_{k}\right)-\beta \lambda_{k}\left[f\left(x_{k}\right)-f^{*}\right]+\lambda_{k} \rho_{k}^{f}+\mu_{k} \rho_{k}^{g} .
\end{aligned}
$$

Assim, se $\overline{\rho_{k}}:=\max \left\{\rho_{k}^{\phi} ; \rho_{k}^{g}\right\}$, temos que independentemente se $x$ é um elemento de $C$ ou não, segue que

$$
d_{X^{*}}^{2}\left(x_{k+1}\right) \leq d_{X^{*}}^{2}\left(x_{k}\right)-\beta \lambda_{k}\left[f\left(x_{k}\right)-f^{*}\right]+\lambda_{k} \rho_{k}^{f}+\mu_{k} \overline{\rho_{k}}
$$

Agora, seja $\delta>0$ um número real arbitrário. Vamos assim, escolher $k_{0}$ tal que para todo $k>k_{0}$, temos

$$
d_{X}\left(x_{k}\right)<\frac{\delta}{W}, \quad \frac{\mu_{k}}{\lambda_{k}} \overline{\rho_{k}}<\frac{\delta}{3}, \quad \rho_{k}^{f}<\frac{\delta}{3} \quad \text { e } \quad\left(\lambda_{k}+\mu_{k}\right) \gamma<\delta
$$

Desta forma, vamos considerar 2 casos: 
1. $d_{X^{*}}\left(\mathscr{P}_{X}\left(x_{k}\right)\right)>d_{X}^{f}(\boldsymbol{\delta})$

2. $d_{X^{*}}\left(\mathscr{P}_{X}\left(x_{k}\right)\right) \leq d_{X}^{f}(\boldsymbol{\delta})$

Vamos supor que o caso 1 ocorra. Desta forma, pela limitação de $\partial f\left(\mathscr{P}_{X}\left(x_{k}\right)\right)$, obtemos que $f\left(x_{k}\right) \geq f\left(\mathscr{P}_{X}\left(x_{k}\right)\right)-W d_{X}\left(x_{k}\right)>f^{*}+\delta$. Assim, por (2.18) e (2.19), obtemos

$$
\begin{aligned}
\frac{d_{X^{*}}^{2}\left(x_{k+1}\right)}{\lambda_{k}} & \leq \frac{d_{X^{*}}^{2}\left(x_{k}\right)}{\lambda_{k}}-\beta\left[f\left(x_{k}\right)-f^{*}\right]+\rho_{k}^{f}+\frac{\mu_{k}}{\lambda_{k}} \overline{\rho_{k}} \\
& <\frac{d_{X^{*}}^{2}\left(x_{k}\right)}{\lambda_{k}}-\delta+\frac{\delta}{3}+\frac{\delta}{3} \\
& <\frac{d_{X^{*}}^{2}\left(x_{k}\right)}{\lambda_{k}}-\frac{\delta}{3}
\end{aligned}
$$

Portanto, podemos concluir que

$$
d_{X^{*}}^{2}\left(x_{k+1}\right)<d_{X^{*}}^{2}\left(x_{k}\right)-\lambda_{k} \frac{\delta}{3}
$$

Agora, vamos supor que o caso 2 seja válido. Assim, pela Propriedade 3 dos operadores $\mathscr{O}_{f}$, $\mathscr{O}_{g}$ e $\mathscr{O}_{\phi}$, temos

$$
\begin{aligned}
d_{X^{*}}\left(x_{k+1}\right) & \leq\left\|x_{k+1}-\mathscr{P}_{X^{*}}\left(x_{k}\right)\right\| \\
& \leq\left\|x_{k+1}-x_{k+1 / 2}\right\|+\left\|x_{k+1 / 2}-x_{k}\right\|+\left\|x_{k}-\mathscr{P}_{X^{*}}\left(x_{k}\right)\right\| \\
& \leq\left(\lambda_{k}+\mu_{k}\right) \gamma+d_{X^{*}}\left(x_{k}\right) \\
& <\delta+d_{X^{*}}\left(x_{k}\right)
\end{aligned}
$$

Portanto, usando o Lema 2.4, segue:

$$
\begin{aligned}
d_{X^{*}}\left(x_{k+1}\right) & \leq d_{X^{*}}\left(\mathscr{P}_{X}\left(x_{k}\right)\right)+2 d_{X}\left(x_{k}\right)+\delta \\
& \leq d_{X}^{f}(\delta)+2 d_{X}\left(x_{k}\right)+\delta \\
& <d_{X}^{f}(\delta)+2 \frac{\delta}{M}+\delta .
\end{aligned}
$$

Logo, por (2.21) e (2.23) e como $\sum_{k} \lambda_{k}=\infty$, segue que existe um $\bar{k}$ suficientemente grande tal que para todo $k \geq \bar{k}$, temos

$$
d_{X^{*}}\left(x_{k}\right)<d_{X}^{f}(\delta)+2 \frac{\delta}{M}+\delta .
$$

Portanto, uma vez que $\delta>0$ é arbitrário e $\lim _{\delta \rightarrow 0^{+}} d_{X}^{f}(\delta)=0$, segue que

$$
\lim _{k \rightarrow \infty} d_{X^{*}}\left(x_{k}\right)=0 \text {. }
$$


Teorema 2.3 Suponha que $\left\{x_{k}\right\}$ é uma sequência gerada pelo Algoritmo II, o operador $\mathscr{O}_{\phi}$ satisfaz as Propriedades 1-2 e $\mathscr{O}_{g}$ e $\mathscr{O}_{f}$ satisfazem as Propriedades 1-3. Ademais, suponha que X é um conjunto compacto e convexo, $C$ satisfaz a Condição de Slater, $\left\{d_{X}\left(x_{k}\right)\right\}$ é limitado, $\mu_{k} \rho_{k}^{g} / \lambda_{k} \rightarrow 0$, $\mu_{k} \rho_{k}^{\phi} / \lambda_{k} \rightarrow 0$ e os tamanhos de passos satisfaçam: $\lambda_{k}, \mu_{k} \rightarrow 0^{+}, \sum \lambda_{k}=\infty, \sum \mu_{k}=\infty$ e $\lambda_{k} / \mu_{k} \rightarrow 0$. Por último, suponha que para todo $z \in\left\{\mathscr{P}_{X}\left(x_{k}\right)\right\}$ existe $v \in \partial f(z)$ com $\|v\| \leq W$, para algum $W \in \mathbb{R}$ fixado. Então

$$
\lim _{n \rightarrow \infty} d_{X^{*}}\left(x_{k}\right)=0
$$

Prova. Segue de imediato dos Lemas 2.8 e 2.9.

Desta forma, fica provada a convergência do Algoritmo II. Infelizmente, aqui não podemos eliminar a hipótese de limitação do conjunto $X$, uma vez que esta se faz necessária para a garantia de convergência dos conjuntos $X_{n}$ e $X_{g_{n}}$ ao conjunto $X$. Podemos ainda fazer uma consideração sobre a Condição de Slater necessária neste novo algoritmo. Notemos que quando a Condição de Slater não é satisfeita, não podemos garantir que uma iteração do Algoritmo I estará contida no conjunto $C$. Desta forma, basta que façamos $C:=\left\{x \in \mathbb{R}^{n} \mid \phi(x)<\varepsilon\right\}$ para algum $\varepsilon>0$ pequeno, para que de forma prática, o Algoritmo II funcione da mesma forma.

\subsection{Relação com Métodos Existentes}

Vamos definir, para os casos em que $C$ é um conjunto simples para projeção, o seguinte algoritmo:

$$
\begin{aligned}
x_{k+1 / 3} & =x_{k}-\lambda_{k} \tilde{\nabla} f\left(x_{k}\right), \quad \tilde{\nabla} f\left(x_{k}\right) \in \partial f\left(x_{k}\right) ; \\
x_{k+2 / 3} & =x_{k+1 / 3}-\mu_{k} \tilde{\nabla} g\left(x_{k}\right), \quad \tilde{\nabla} g\left(x_{k}\right) \in \partial g\left(x_{k}\right) ; \\
x_{k+1} & =\mathscr{P}_{C}\left(x_{k+2 / 3}\right) .
\end{aligned}
$$

Logo, este método é apenas uma versão particular do Algoritmo I, bastando fazer o cálculo do subgradiente da função $g$ em $x_{k}$ e usando a projeção como operador de factibilidade. O leitor pode ficar apreensivo com relação a avaliação do subgradiente da função $g$ ser em um ponto diferente do que foi feito nas provas de convergência, entretanto se observamos que

$$
\begin{aligned}
\left\|x_{k+2 / 3}-y\right\|^{2} \leq & \left\|x_{k+1 / 3}-y\right\|^{2}-\mu_{k}\left(g\left(x_{k}\right)-g(y)\right)+\mu_{k}^{2}\left\|\tilde{\nabla} g\left(x_{k}\right)\right\|^{2} \\
= & \left\|x_{k+1 / 3}-y\right\|^{2}-\mu_{k}\left(g\left(x_{k+1 / 3}\right)-g(y)\right)+\mu_{k}^{2}\left\|\tilde{\nabla} g\left(x_{k}\right)\right\|^{2}+\mu_{k}\left(g\left(x_{k+1 / 3}\right)-g\left(x_{k}\right)\right) \\
\leq & \left\|x_{k+1 / 3}-y\right\|^{2}-\mu_{k}\left(g\left(x_{k+1 / 3}\right)-g(y)\right)+\mu_{k}^{2}\left\|\tilde{\nabla} g\left(x_{k}\right)\right\|^{2} \\
& \quad+\mu_{k} \lambda_{k}\left\|\tilde{\nabla} g\left(x_{k+1 / 3}\right)\right\|\left\|\tilde{\nabla} f\left(x_{k}\right)\right\|,
\end{aligned}
$$


temos a certeza que o Algoritmo I converge sob tais circunstâncias. De modo análogo, podemos enxergar este método como uma versão particular do Algoritmo II, sendo que neste caso, nenhum subgradiente da função $\phi$ será utilizado durante a execução do método.

Podemos ainda escrever o algoritmo (2.24) de forma mais simples

$$
x_{k+1}=\mathscr{P}_{C}\left(x_{k}+\mu_{k} \tilde{\nabla} F_{\sigma_{k}}\left(x_{k}\right)\right) \text {, }
$$

sendo $F_{\sigma}(x):=\sigma f(x)+g(x)$ e $\sigma_{k}=\lambda_{k} / \mu_{k}$. Portanto, para os casos em que $C$ é um conjunto simples, temos que os nossos algoritmos abrangem os métodos desenvolvidos em [10,11], mas com a vantagem de não requererem nenhum tipo de busca linear ou técnicas de bundle. Ademais, os algoritmos citados nas últimas duas seções, conseguem lidar com conjuntos mais sofisticados que os trabalhos supracitados.

\subsection{Operadores de Otimalidade Aplicáveis}

Apresentamos nesta seção operadores que obedecem as propriedades 1-3 exigidas por ambos os algoritmos. É importante notar que as propriedades por nós exigidas são as mesmas que as apresentadas em [8], e portanto, é razoável pensar que todos os operadores aplicáveis deste trabalho sejam também operadores para os nossos métodos. De fato, apresentamos dois resultados que comprovam que os algoritmos (1.3) e (1.4) são operadores que satisfazem todas as propriedades requeridas, todavia, omitimos as provas de ambas as proposições, uma vez que as demonstrações são imediatas de [4, Lemma 2.1] e [8, Proposition 3.2].

Proposição 2.5 Suponha a seguinte limitação dos subgradientes:

$$
\|v\| \leq K_{l}, \quad \forall v \in \partial f_{l}\left(x_{k}\right) \cup \partial f_{l}\left(x_{k, l-1}\right), \quad l=1, \ldots, m, \quad k=0,1, \ldots
$$

Então as iterações de (1.3) satisfazem a Propriedade 1 com $\rho_{k} \leq \lambda_{k}\left(\sum_{l=1}^{m} K_{l}\right)^{2}$ e $\beta=2$, e a Propriedade 3 com $\gamma=\sum_{l=1}^{m} K_{l}$.

Proposição 2.6 Suponha que (2.25) seja válido. Então as iterações de (1.4) satisfazem a Propriedade $1 \operatorname{com} \beta=2 e$

$$
\rho_{k}=(4+1 / m) \bar{\lambda}_{k} K^{2}+4 K\left(2 d_{C}\left(x_{k-1 / 3}\right)+\mu_{k-1} \gamma\right),
$$

sendo $K:=\sum_{l=1}^{m} K_{l}$ e $\bar{\lambda}_{k}:=\max \left\{\lambda_{k}, \lambda_{k-1}\right\}$ para $k>0$. A Propriedade 3 também é satisfeita com $\gamma=K$. 
Aqui vale observar que o termo de erro $\rho_{k}$ é diferente do apresentado em [8], uma vez que devemos considerar um passo adicional entre duas sucessivas aplicações do operador de subgradiente incremental agregado, porém, como dito anteriormente, o resultado é imediato da prova encontrada em [8, Proposition 3.2].

O resultado [6, Proposição 3] garante ainda que os algoritmos (1.5)-(1.8) cumprem as exigências das Propriedades 1-3. Ademais, como pode ser visto adiante, métodos baseados em $\varepsilon$ subgradientes também podem ser utilizados.

Definição 2.4 Dado $\varepsilon>0$, o $\varepsilon$-subdiferencial de uma função $f$ em um determinado ponto $x$ é definido como sendo o conjunto $\partial_{\varepsilon} f(x):=\{v \mid f(x)+\langle v, y-x\rangle-\varepsilon \leq f(y), \forall y\}$. Todo elemento pertencente a $\partial_{\varepsilon} f(x)$ é dito ser um $\varepsilon$-subgradiente de $f$ em $x$.

Proposição 2.7 Seja $f: \mathbb{R}^{n} \rightarrow \mathbb{R}$ uma função convexa. Dado $x_{0} \in \mathbb{R}^{n}$, definimos uma iteração $k$ como

$$
x_{k+1}:=x_{k}-\lambda_{k} v_{k},
$$

sendo $v_{k} \in \partial_{\varepsilon_{k}} f\left(x_{k}\right) e\left\|v_{k}\right\|<C$, para algum $C>0$. Desta forma, se $\varepsilon_{k} \rightarrow 0$, as iterações (2.26) satisfazem as Propriedades 1-3. Ademais, se $\varepsilon<R \lambda_{k}$ para algum $R>0$, então $\rho_{k}^{f}=O\left(\lambda_{k}\right)$.

Prova. Observemos que para qualquer $x \in C$ temos

$$
\begin{aligned}
\left\|x_{k+1}-x\right\|^{2} & =\left\|x_{k}-\lambda_{k} v_{k}-x\right\|^{2} \\
& \leq\left\|x_{k}-x\right\|^{2}-2 \lambda_{k}\left\langle v_{k}, x_{k}-x\right\rangle+\lambda_{k}^{2}\left\|v_{k}\right\|^{2} \\
& \leq\left\|x_{k}-x\right\|^{2}-2 \lambda_{k}\left(f\left(x_{k}\right)-f(x)+\varepsilon\right)+\lambda_{k}^{2} C^{2} \\
& =\left\|x_{k}-x\right\|^{2}-2 \lambda_{k}\left(f\left(x_{k}\right)-f(x)\right)+\lambda_{k} \rho_{k}^{f},
\end{aligned}
$$

sendo $\rho_{k}^{f}=\lambda_{k} C^{2}+2 \varepsilon$. Ademais, temos que

$$
\left\|x_{k+1}-x_{k}\right\|=\lambda_{k}\left\|v_{k}\right\| \leq \lambda_{k} C \text {. }
$$

Portanto, se $\lambda_{k} \rightarrow 0^{+}$, segue o resultado.

Finalmente, na próxima seção, apresentamos um operador de factibilidade concreto para o Algoritmo I. 


\subsection{Um Operador de Factibilidade Aplicável}

Vamos inicialmente mostrar que a inequação (2.4) da propriedade do operador de factibilidade pode ser verificada sem que haja a necessidade do cálculo da distância do iterando $x_{k}$ sobre o conjunto $C$. De fato, vamos considerar o seguinte resultado que pode ser encontrado em [15]:

Proposição 2.8 Sejam X um espaço munido de produto interno e $C_{1}, \ldots, C_{p}$ subconjuntos convexos de X. Suponha que exista $D>0$ tal que $\sup _{x, y \in C_{i}} d(x, y)<D$ para todo $i=1, \ldots$, m e exista $\rho>0$ tal que

$$
\rho<\sup _{x \in C} d\left(x, X \backslash \bigcap_{i=1}^{p-1} C_{i}\right)
$$

sendo $C=\bigcap_{i=1}^{p} C_{i}$. Então, se $d_{C_{i}}(x) \leq \varepsilon, i=1, \ldots, m$, segue que

$$
d_{C}(x) \leq \frac{2\left(\frac{D-\rho}{\rho}\right)^{p+1}-\left(\frac{D-\rho}{\rho}\right)^{p}-\left(\frac{D-\rho}{\rho}\right)}{\left(\frac{D-\rho}{\rho}\right)-1} \varepsilon
$$

Com este resultado em mãos, podemos enunciar uma proposição que garante a existência de um algoritmo para o operador de factibilidade.

Proposição 2.9 Considere as funções convexas $h_{i}$ que caracterizam $C$ em (1.13) e AC $\mathbb{R}^{n}$ um conjunto convexo e compacto. Suponha que existem $\bar{E}>0$ e $\alpha>0$ tal que

$$
d_{\operatorname{lev}_{0}\left(h_{i}\right)}(x) \leq \bar{E} h_{i}(x)^{\alpha}, \quad i=1, \ldots, p, \quad \forall x \in A \backslash C_{i}
$$

Ainda, suponha que exista $\rho>0$ tal que

$$
\rho<\sup _{x \in A \cap C} d\left(x, \mathbb{R}^{n} \backslash \bigcap_{i=1}^{p-1}\left[A \cap \operatorname{lev}_{0}\left(h_{i}\right)\right]\right) .
$$

Então, dado $x_{0} \in \mathbb{R}^{n}$, o seguinte algoritmo é um operador de factibilidade para o Algoritmo I:

$$
\begin{aligned}
& x=x_{0} \\
& \text { Enquanto }\left(\max _{1 \leq i \leq p} h_{i}(x)>\mu_{k}^{\varepsilon / \alpha}\right) \\
& \quad x=\mathscr{F}_{C}(x)
\end{aligned}
$$


sendo $\varepsilon>0$ um número real fixado e

$$
\mathscr{F}_{C}(x)= \begin{cases}x-\kappa \frac{\phi(x)}{\|v\|^{2}} v & \text { se } x \notin C \\ x & \text { se } x \in C\end{cases}
$$

tal que $\kappa \in(\Lambda, 2-\Lambda)$ para algum $\Lambda \in(0,1), \phi(x)=\max _{1 \leq i \leq p} h_{i}(x) e v \in \partial \phi(x)$.

Prova. Vamos mostrar primeiramente que para todo $x \in \mathbb{R}^{n}$ e $y \in C$, temos que

$$
\left\|\mathscr{F}_{C}(x)-y\right\|^{2} \leq\|x-y\|^{2} .
$$

De fato, caso $x \in A \cap C$, então pela própria definição de $\mathscr{F}_{C}(x)$ segue de imediato o resultado. Agora, suponha que $x \notin A \cap C$. Então, temos que

$$
\begin{aligned}
\left\|\mathscr{F}_{C}(x)-y\right\|^{2} & =\|x-y\|^{2}-2 \kappa \frac{\phi(x)}{\|v\|^{2}}\langle v, x-y\rangle+\kappa^{2} \frac{\phi(x)^{2}}{\|v\|^{2}} \\
& \leq\|x-y\|^{2}-2 \kappa \frac{\phi(x)}{\|v\|^{2}}[\phi(x)-\phi(y)]+\kappa^{2} \frac{\phi(x)^{2}}{\|v\|^{2}} \\
& =\|x-y\|^{2}-(2-\kappa) \kappa \frac{\phi^{2}(x)}{\|v\|^{2}}
\end{aligned}
$$

Portanto, o resultado é válido. Agora, provemos que existem $E>0$ e $\varepsilon>0$ fixados tal que

$$
d_{C}(\bar{x}) \leq E \mu_{k}^{\varepsilon}
$$

sendo $\bar{x}$ a última iteração do algoritmo (2.30). De fato, uma vez que (2.28) é válido, segue de imediato que

$$
d_{A \cap \operatorname{lev}_{0}\left(h_{i}\right)}(x) \leq \bar{E}\left[\max _{1 \leq i \leq p} h_{i}(x)\right]^{\alpha}, \quad \forall x \in A \backslash C .
$$

Além disso, todas as hipóteses da Proposição 2.8 são satisfeitas, uma vez que a condição (2.29) é válida e os conjuntos $A \cap \operatorname{lev}_{0}\left(h_{i}\right)$ são todos limitados. Desta forma, como para todo $x \in A$ temos que $d_{A \cap C}(x)=d_{C}(x)$ e $d_{A \cap \operatorname{lev}_{0}\left(h_{i}\right)}(x)=d_{\operatorname{lev}_{0}\left(h_{i}\right)}(x)$, temos que

$$
d_{C}(x) \leq E\left[\max _{1 \leq i \leq p} h_{i}(x)\right]^{\alpha}, \quad \forall x \in A \backslash C,
$$

sendo $E=\frac{2\left(\frac{D-\rho}{\rho}\right)^{n+1}-\left(\frac{D-\rho}{\rho}\right)^{n}-\left(\frac{D-\rho}{\rho}\right)}{E} \bar{E}$. Portanto, como exigimos que

$$
\max _{1 \leq i \leq p} h_{i}(\bar{x}) \leq \mu_{k}^{\varepsilon / \alpha},
$$

segue de imediato que

$$
d_{C}(\bar{x}) \leq E \mu_{k}^{\varepsilon} .
$$


Antes de prosseguirmos, façamos algumas observações pertinentes. Primeiro, notemos que se supormos a sequência $\left\{x_{k}\right\}$ gerada pelo Algoritmo I limitada, segue de imediato da Propriedade 3 que $\left\{x_{k+1 / 3}\right\}$ e $\left\{x_{k+2 / 3}\right\}$ são sequências limitadas. Consequentemente, $\left\{\mathscr{P}_{\operatorname{lev}_{0}\left(h_{i}\right)}\left(x_{k+2 / 3}\right)\right\}$ é também limitada para todo $i=1, \ldots, p$. Desta forma, não é absurdo supormos que exista um conjunto $A$ convexo e compacto de tal maneira que englobe as sequências $\left\{x_{k+2 / 3}\right\}$ e $\left\{\mathscr{P}_{\operatorname{lev}_{0}\left(h_{i}\right)}\left(x_{k+2 / 3}\right)\right\}$. Ademais, notemos que o operador $\mathscr{F}_{C}$ pode ser definido de outras formas (ver [8]) e ainda satisfazer a propriedades de não-expansividade requerida $\left(\left\|\mathscr{F}_{C}(x)-y\right\| \leq\|x-y\|\right)$.

Apontamos ainda que a exigência (2.29) é satisfeita para muitos conjuntos, por exemplo, quando int $C \neq \emptyset$ :

Propriedade 5 Sejam $X$ um espaço munido de produto interno e $C_{1}, \ldots, C_{p}$ subconjuntos convexos de X. Se C $=\bigcap C_{i}$ tem interior não-vazio, então a condição (2.29) é satisfeita.

Prova. Uma vez que $C$ é definido como sendo a interseção dos conjuntos $C_{i}$, segue de imediato que

$$
C \cap\left(X \backslash \bigcap_{i=1}^{p-1} C_{i}\right)=\emptyset .
$$

Portanto, uma vez que int $C \neq \emptyset$, segue que existem $c \in C$ e $r>0$ tal que

$$
B(c, r) \cap\left(X \backslash \bigcap_{i=1}^{p-1} C_{i}\right)=\emptyset .
$$

Por conseguinte, o resultado segue, já que

$$
r<\sup _{x \in C} d\left(x, X \backslash \bigcap_{i=1}^{p-1} C_{i}\right) .
$$

Entretanto, ressaltamos que int $C \neq \emptyset$ não é uma condição necessária para que (2.29) seja satisfeita (ver Figura 2.1).

Finalmente, mostremos que é possível obter (2.28) para vários tipos de funções convexas. De fato, exibimos abaixo uma definição e dois teoremas que podem ser encontrados em [1] que serão de grande valia para o nosso objetivo de construir um operador de factibilidade.

Definição 2.5 Uma direção $s \in \mathbb{R}^{n}$ é dita ser normal a $C \subset \mathbb{R}^{n}$ em $x$ se

$$
\langle s, y-x\rangle \leq 0, \quad \forall y \in C
$$




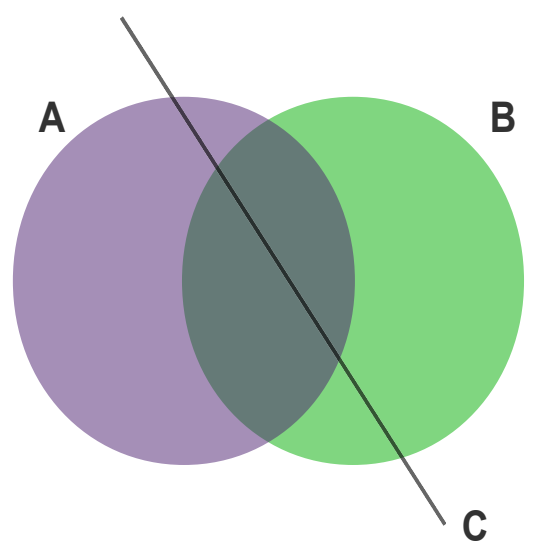

Figura 2.1: Ilustração de interseção de 3 conjuntos que satisfaz (2.29), mas possui interior vazio.

O conjunto de todas estas direções é chamado de cone normal a $C$ em x e denotado por $N_{C}(x)$.

Teorema 2.4 Seja $C \subset \mathbb{R}^{n}$ um conjunto não-vazio, fechado e convexo. Dado um ponto $x \in \mathbb{R}^{n}$, um elemento $p \in C$ é a projeção de $x$ em $C$ se, e somente se,

$$
\langle x-p, y-p\rangle \leq 0, \quad \forall y \in C .
$$

Teorema 2.5 Seja $f: \mathbb{R}^{n} \rightarrow \mathbb{R}$ uma função convexa e suponha que $0 \notin \partial f(x)$, então

$$
N_{\operatorname{lev}_{f(x)}(f)}=\mathbb{R}^{+} \partial f(x)
$$

Por conseguinte, podemos garantir a majoração de $d_{\operatorname{lev}_{0}\left(h_{i}\right)}(x), 1 \leq i \leq p$, para várias situações. Mais especificamente, consideremos o resultado abaixo:

Proposição 2.10 Sejam $h: \mathbb{R}^{n} \rightarrow \mathbb{R}$ uma função convexa e $H:=\operatorname{lev}_{0}(h)$. Suponha que para todo $v \in \partial h(x) \operatorname{com} x \in \partial H$ temos

$$
\|v\| \geq V, \quad \text { para algum } V>0
$$

Então existe $\bar{E}>0$ tal que

$$
d_{H}(x) \leq \bar{E} h(x), \quad \forall x \in \mathbb{R}^{n} \backslash H .
$$

Prova. Observemos inicialmente que para qualquer $x \in \mathbb{R}^{n} \backslash H$ temos que $h\left(\mathscr{P}_{H}(x)\right)=0$. Portanto, $y \in H \Leftrightarrow y \in \operatorname{lev}_{h\left(\mathscr{P}_{H}(x)\right)}(h)$, e desta forma, pelo Teorema 2.4, segue que

$$
\left\langle x-\mathscr{P}_{H}(x), y-\mathscr{P}_{H}(x)\right\rangle \leq 0, \quad \forall y \in \operatorname{lev}_{h\left(\mathscr{P}_{H}(x)\right)}(h)
$$


Logo, segue imediatamente da definição de cone normal que $x-\mathscr{P}_{H}(x) \in N_{\operatorname{lev}_{h\left(\mathscr{P}_{H}(x)\right)}(h)}\left(\mathscr{P}_{H}(x)\right)$, e portanto, pelo Teorema 2.5, segue que existe $v \in \partial h\left(\mathscr{P}_{H}(x)\right)$ e $t>0$ tal que $x-\mathscr{P}_{H}(x)=t v$. Portanto, por definição de subgradiente, segue que

$$
\left\langle v, x-\mathscr{P}_{H}(x)\right\rangle \leq h(x)-h\left(\mathscr{P}_{H}(x)\right) \Rightarrow\|v\| d_{H}(x) \leq h(x) \Rightarrow d_{H}(x) \leq \frac{1}{V}[h(x)] .
$$

Assim, definindo $\bar{E}=1 / V$ segue que

$$
d_{H}(x) \leq \bar{E} h(x), \quad \forall x \in \mathbb{R}^{n} \backslash H
$$

Destacamos que a necessidade que a condição (2.33) seja válida na Proposição 2.10 é uma premissa mais fraca que a Condição de Slater exigida no Algoritmo II, uma vez que aqui pedimos apenas que exista $x_{i} \in \mathbb{R}^{n}$ tal que $h_{i}\left(x_{i}\right)<0$, sem que este $x_{i}$ seja o mesmo para todo $i$. 


\section{Tomografia e Métodos de Reconstrução de Imagens}

Com a finalidade de tornar a aplicação do nosso método compreensível, fazemos uma introdução à tomografia computadorizada, descrevendo o seu funcionamento e apresentado alguns dos métodos mais conhecidos de reconstrução de imagens tomográficas.

\subsection{Tomografia}

Derivada de dois termos gregos, tomos (corte) e grafein (escrever; registrar), a tomografia nada mais é que a obtenção de uma imagem secional de um determinado objeto de maneira não destrutiva. Esta imagem pode ser obtida de várias formas (por raios-x, ressonância magnética, ultrassom, etc) e para numerosos fins [16-18].

\subsubsection{Tomografia por Transmissão}

A imagem radiográfica foi a primeira utilização prática dos raios-x. Seu funcionamento baseiase na propriedade da radiação atravessar a matéria de maneira distinta para os tecidos do paciente. Quanto maior a taxa de absorção do tecido, mais radiação é retida e consequentemente, a detecção desta é reduzida (ver Figura 3.1). Os ossos são bons exemplos de tecidos que podem ser estudados com facilidade dessa maneira, já que absorvem muito mais a radiação do que tecidos mais macios, como os músculos. A dificuldade inerente a esta técnica é a obtenção de uma boa imagem de um objeto com pouca taxa de absorção rodeado de outros com maior taxa.

A solução encontrada foi obter, a partir de vários ângulos distintos, várias projeções radiográficas e, através de um modelo matemático, reconstruir a taxa de atenuação de todos os pontos do interior do paciente. Esta técnica é conhecida como tomografia computadorizada ou simplesmente TC. 


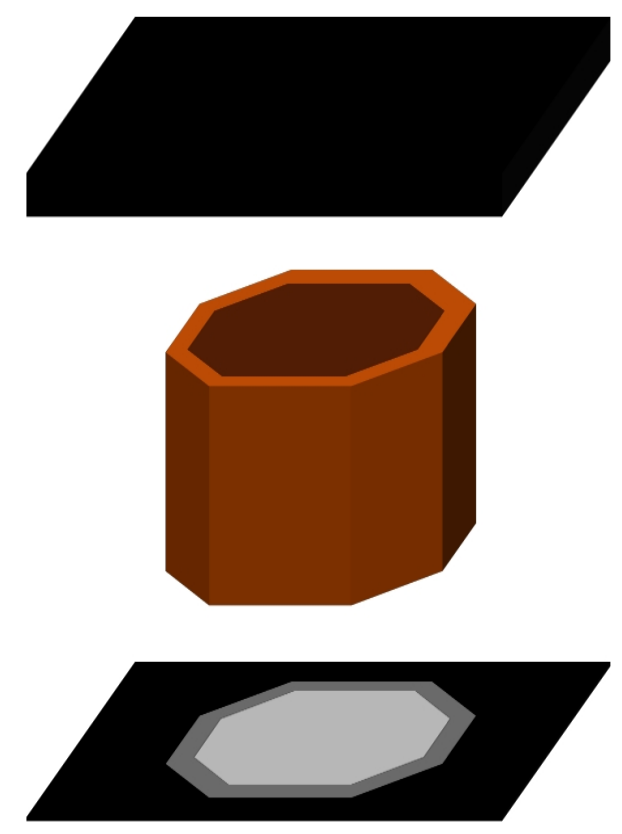

Figura 3.1: Ilustração de uma radiografia. Na parte superior encontra-se a fonte de raios x e abaixo temos o filme detector.

\subsubsection{Tomografia por Emissão}

Além da obtenção de uma imagem por raios-x (Tomografia por Transmissão), onde a preocupação essencial é reconstruir a constituição física de um determinado objeto, existe também a chamada Tomografia por Emissão. O objetivo central é entender um processo fisiológico por meio de um marcador radioativo ministrado ao paciente.

Existem dois processos bem conhecidos: Tomografia por Emissão de Pósitrons (PET) e Tomografia Computadorizada por Emissão de Fóton Único (SPECT) . No primeiro, o marcador é artificial e decai emitindo pósitrons, os quais colidem com elétrons e geram dois fótons que percorrem direções opostas. Estes, por sua vez, são recebidos por um arranjo de detectores. Cada par de detectores determina uma reta e estas contribuem na reconstrução da imagem final (ver Figura 3.2).

Já no caso do SPECT, o marcador é um isótopo natural que durante o processo de decaimento produz um único fóton, o qual é detectado por câmeras posicionadas em torno do paciente (ver Figura 3.3). Neste processo ocorre a absorção de alguns fótons pelos colimadores presentes na câmera, uma vez que estes fotóns não chegaram de uma direção previamente estipulada, gerando por consequência, uma imagem menos rica que a produzida por PET.

Independentemente de qual processo tomográfico é escolhido para a reconstrução do interior do 


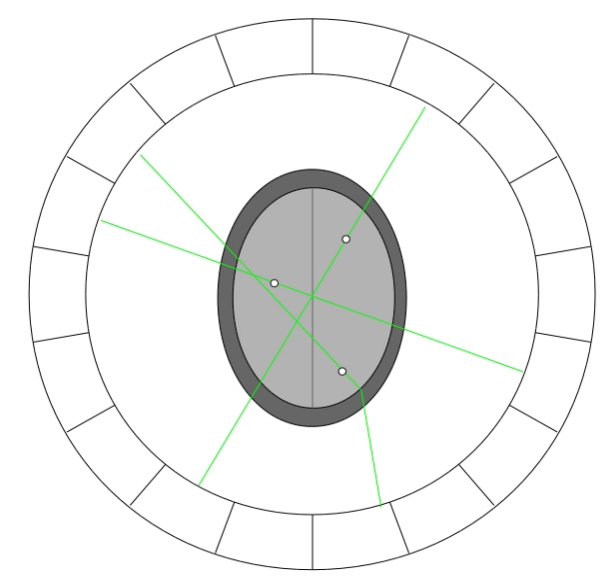

Figura 3.2: Ilustração de uma tomografia por emissão de pósitrons. Os traços em verde são as retas detectadas pelos pares de detectores em direções opostas.

paciente, o problema da reconstrução da imagem é inevitavelmente mal-posto. Na seção seguinte apresentamos uma definição formal de um problema mal-posto.

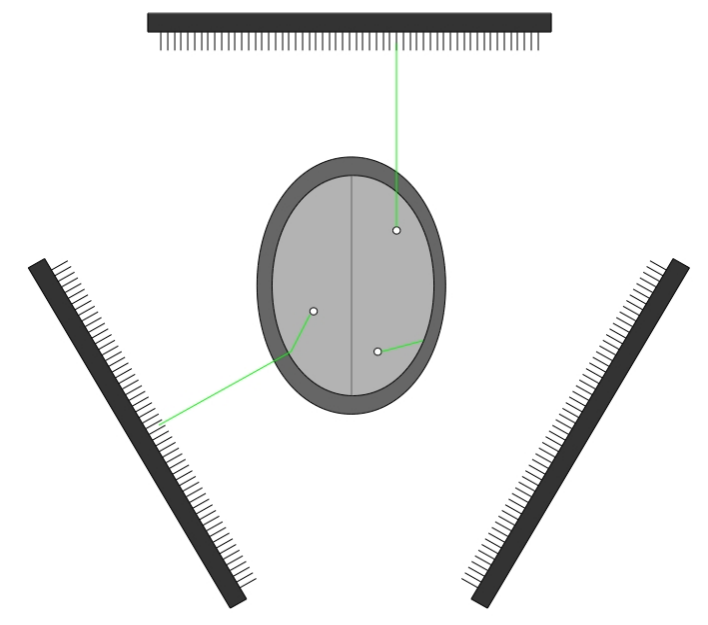

Figura 3.3: Ilustração de uma tomografia por emissão de fóton único. Os traços em verde são os caminhos percorridos pelos fótons. Os três aparatos são os detectores com colimadores.

\subsubsection{Modelagem Matemática Contínua}

Vamos considerar o problema de determinar a taxa de atenuação de radiação em cada ponto $\left(x_{1}, x_{2}\right)$ do interior do paciente. Desejamos encontrar, a partir da soma das taxas de atenuação ao longo de um feixe de radiação $\mathrm{L}$, uma função $f: \Omega \subset \mathbb{R}^{2} \rightarrow \mathbb{R}$ de tal maneira que $f(x)$ indique a taxa de atenuação no ponto $x$. Olhando para o problema de forma contínua, isso nada mais é que determinar $f$ a partir da seguinte integral de linha (ver Figura 3.4), conhecida como Transformada 
de Radon (TR):

$$
R[f](\theta, t):=\int_{\mathbb{R}} f(t \cos (\theta)-s \sin (\theta), t \sin (\theta)+s \cos (\theta)) d s
$$

Quando não houver dúvida sobre qual função estamos aplicando a TR, podemos denotar $p_{\theta}(t):=$ $R[f](\theta, t)$.

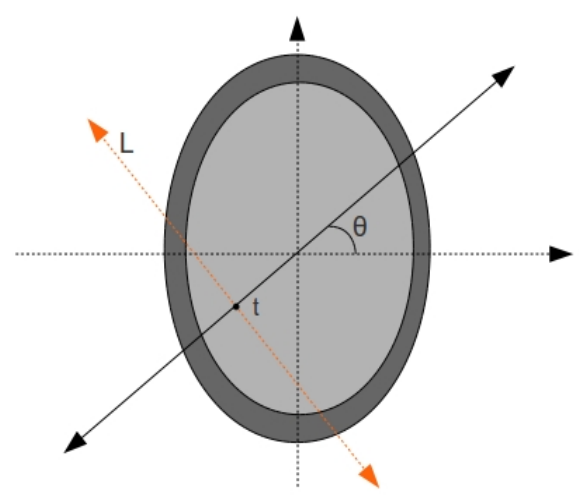

Figura 3.4: Ilustração de um feixe de radiação (linha tracejada laranja) atravessando um objeto com taxas de atenuação distintas ao longo do trajeto $\mathrm{L}$.

Como consequência da formalização do problema, percebemos que se conseguirmos inverter a TR, teremos por conseguinte, a função $f$ desejada. Felizmente, um resultado conhecido como Teorema da Fatia de Fourier, permite-nos obter a inversa da TR. Para que a compreensão do resultado seja facilitada, apresentamos a definição da Transformada de Fourier $\mathscr{F}$ antes de expormos o teorema.

Definição 3.1 Dada uma função $f: \mathbb{R}^{n} \rightarrow \mathbb{C}$, definimos respectivamente a sua Transformada $e$ Inversa da Transformada de Fourier como:

$$
\begin{gathered}
\mathscr{F}[f](x):=\hat{f}=\int_{\mathbb{R}^{n}} f(s) e^{-i\langle x, s\rangle} d s \\
\mathscr{F}^{-1}[f](x):=\frac{1}{(2 \pi)^{n}} \int_{\mathbb{R}^{n}} f(x) e^{i\langle s, x\rangle} d x .
\end{gathered}
$$

Teorema 3.1 (Teorema da Fatia de Fourier) Seja $f: \mathbb{R}^{2} \rightarrow \mathbb{C} \in L_{\mathbb{R}^{n}}^{1}$, então

$$
\hat{p}_{\theta}(s)=\hat{f}(s \cos \theta, s \sin \theta) .
$$

Portanto, operando com a Transformada Inversa de Fourier apropriada, obtemos pela equação (3.2) a função $f$ desejada. Contudo, como na prática é inviável o conhecimento da TR para todo par $(\theta, t)$, a aplicação do Teorema 3.1 puramente não resolve o problema da reconstrução da imagem. 
Para o leitor interessado, deixamos aqui uma referência para o método conhecido como Retroprojeção Filtrada [16], o qual utiliza-se de fórmulas de inversão convenientes baseadas no Teorema da Fatia de Fourier para o seu funcionamento.

Infelizmente, métodos baseados na inversão da TR não apresentam uma boa recuperação, uma vez que o problema de reconstrução de imagens tomográficas é um problema mal-posto [19], ou seja, ao menos uma das seguintes afirmações é falsa:

- Para todo conjunto de dados existe uma solução;

- A solução sempre é única;

- A solução depende continuamente dos dados.

Como solução para este inconveniente, vários métodos iterativos têm se mostrado promissores na execução desta tarefa. Na subseção seguinte, fazemos a discretização do problema (3.1) com o intuito de introduzirmos posteriormente os métodos iterativos mais conhecidos na tomografia.

\subsubsection{Modelagem Matemática Discreta}

Suponha agora que $\Omega$ é um retângulo e está divido em $n$ quadrados (pixels). Simplificaremos o problema e procuraremos por uma função $f$ que seja constante em cada quadrado, ainda que a verdadeira solução não apresente este comportamento (ver Figura 3.5). Com esta discretização do conjunto $\Omega$, podemos compreender, daqui por diante, a TR como uma matriz, já que trabalharemos em espaços de dimensão finita e vale a igualdade $R[\alpha f+\beta g]=\alpha R[f]+\beta R[g]$. Considere ainda que existam $m$ feixes de radiação e que $x_{j}$, para $j=1 \ldots n$, seja a notação para o valor de $f$ em cada um dos quadrados e $b_{i}$ o valor da integral de linha de $f$ ao longo do feixe $i$. Considerando $R \in \mathbb{R}^{m \times n}, x \in \mathbb{R}^{n}, b \in \mathbb{R}^{m}$, podemos interpretar o nosso problema como o seguinte sistema linear:

$$
R x=b .
$$

Note que o elemento $r_{i j}$ da matriz da Transformada de Radon nada mais é que o comprimento da interseção entre o raio $i$ e o pixel $j$.

A discretização do problema ainda revela os seus inconvenientes. A solução nem sempre é única e nem sempre irá existir para todo sistema linear $R x=b$. Além disso, a matriz obtida $R$, é usualmente mal-condicionada, fazendo com que o problema discreto funcione "quase" de maneira descontínua. 


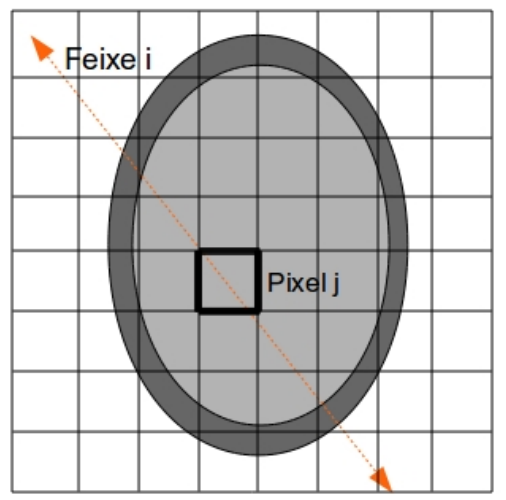

Figura 3.5: Ilustração da divisão por pixels de uma região retangular contendo um feixe de radiação (linha tracejada laranja) atravessando um corpo denso.

\subsection{Métodos}

Dada as propriedades desfavoráveis discutidas anteriormente, buscamos por um método de solução que de alguma forma minimize estas características indesejadas, mas que ao mesmo tempo, respeite os dados obtidos pelo tomógrafo. Como solução, apresentamos o seguinte problema de otimização convexa:

$$
\text { Minimizar : } g(x)+\gamma r(x)
$$

Sujeito a : $x \in X$.

O conjunto $X$ representa algumas informações que possamos ter a priori da solução, como por exemplo $x \in \mathbb{R}_{+}^{n}$. Já a função $g(x)$ força a consistência com os dados obtidos. Para isto, normalmente utilizamos $g(x)=F(R x, b)$, sendo que quando o sistema é consistente, $F(y, b)$ só atinge o seu valor mínimo quando $y=b$. Caso o sistema seja inconsistente, buscamos por $x$ que de alguma forma minimize a discrepância entre $R x$ e $b$. Observemos que, escolhendo $F$ apropriadamente, isto possibilita que exista uma solução razoável quando a mesma não existe no problema original $(R x=b)$. A estabilidade da solução é garantida pela função $r(x)$ e o seu coeficiente $\gamma>0$. A ideia aqui é que $r(x)$ favoreça soluções mais suaves e $\gamma$ controle o quanto que esta regularização terá importância no nosso modelo. Existem estudos que indicam como obter um bom valor para $\gamma$, para saber mais veja [19,20].

Uma vez redefinido o nosso problema, podemos dar continuidade em como reconstruir imagens tomográficas. Faremos uma breve apresentação nas próximas subseções de vários algoritmos que 
se propõem a resolver (3.4) e recomendamos, por ser uma apresentação superficial, que o leitor mais interessado procure mais informações nas referências apresentadas.

\subsubsection{Técnica de Reconstrução Algébrica - ART}

Antes de apresentarmos a Técnica de Reconstrução Algébrica, assumiremos daqui por diante que todas as funções e conjuntos não explicitamente declarados são convexos e fechados. Agora, seguindo a definição de projeção, dados um hiperplano $H=\left\{y \in \mathbb{R}^{n} \mid\langle a, y\rangle=\beta\right\}$ e $x \in \mathbb{R}^{n}$, podemos calcular a projeção de $x$ em $H$ como:

$$
\pi_{H}(x)=x-\frac{(\langle a, x\rangle-\beta)}{\|a\|^{2}} a .
$$

Se em (3.4) definirmos $g(x):=c, r(x):=c$ e $X:=\left\{x \in \mathbb{R}^{n} \mid R x=b\right\} \neq \emptyset$, a solução deve satisfazer $\left\langle R_{[i,:]}, x\right\rangle=b_{i}$, para todo $i$, sendo $R_{[i,:]}$ o vetor vertical da $i$-ésima linha da matriz $R$. Portanto, definindo o hiperplano $S_{i}:=\left\{x \in \mathbb{R}^{n} \mid\left\langle R_{[i,:]}, x\right\rangle=b_{i}\right\}$, temos o seguinte algoritmo:

\section{Algoritmo 1 - ART}

Dados: $x_{o} \in \mathbb{R}^{n} ; b \in \mathbb{R}^{m} ;\left\{\lambda_{k}\right\} \subset[\delta, 2-\delta] \operatorname{com} \delta \in(0,1)$

Enquanto (critério de parada $=$ falso $)\{$

$$
\begin{aligned}
& x_{k, 0}=x_{k} ; \\
& \text { Para }(\mathrm{i}=1 \text { até } \mathrm{i}=\mathrm{m})\{ \\
& \quad x_{k, i}=\left(1-\lambda_{k}\right) x_{k, i-1}+\lambda_{k} \pi_{S_{i}}\left(x_{k, i-1}\right) \\
& \} \\
& x_{k+1}=x_{k, m}
\end{aligned}
$$$$
\} \text {. }
$$

Note que este algoritmo trabalha com relaxação das projeções, uma vez que podemos ficar no meio do caminho da projeção $\left(0<\lambda_{k}<1\right)$ ou eventualmente passarmos do ponto exato $(1<\lambda<2)$. Portanto, quando $\lambda_{k} \equiv 1$ para todo $k$, o algoritmo é uma sequência de projeções exatas sobre os hiperplanos $S_{i}$. A escolha apropriada do parâmetro $\lambda_{k}$, bem como a ordem em que são feitas as projeções, são de grande valia para a rápida convergência do algoritmo [16]. 
A Técnica de Reconstrução Algébrica foi desenvolvida por Kaczmarz e trinta e três anos mais tarde, revista por Gordon [21]. Um problema deste algoritmo é a possibilidade do conjunto $X$ ser vazio, ou seja, no caso em que o sistema linear (3.3) não tem solução o algoritmo é falho. Para contornar este problema, definimos $g(x):=\frac{1}{2} \sum_{i=1}^{n} d_{S_{i}}^{2}(x), r(x):=c$ e $X:=\mathbb{R}^{n}$. Nessas circunstâncias, os resultados [22-24] mostram que o algoritmo apresentado acima converge para a solução ótima do novo problema quando $\lambda_{k} \rightarrow 0^{+} \mathrm{e} \sum_{k=0}^{\infty} \lambda_{k}=\infty$.

\subsubsection{Projeção sobre Convexos - POCS}

A Projeção sobre Convexos é uma generalização do algoritmo ART, uma vez que aqui não trabalhamos apenas com hiperplanos, mas também com qualquer conjunto convexo. Portanto, é natural pensar que se soubermos projetar neste conjunto, a única mudança que teremos no algoritmo ART é a substituição de $\pi_{S_{i}}(x)$ por esta projeção. De fato, a convergência é assegurada com esta modificação [25].

Definindo $g(x):=c, r(x):=c, X:=\bigcap_{i=1}^{m} X_{i} \neq \emptyset, \operatorname{com} X_{i}$ sendo um conjunto convexo para todo $i$ e $\mathscr{P}_{X_{i}}(x)$ a projeção de $x$ no conjunto $X_{i}$, temos o algoritmo:

\section{Algoritmo 2 - POCS}

Dados: $x_{o} \in \mathbb{R}^{n} ; b \in \mathbb{R}^{m} ;\left\{\lambda_{k}\right\} \subset[\delta, 2-\delta] \operatorname{com} \delta \in(0,1)$

Enquanto (critério de parada $=$ falso $)\{$

$$
\begin{aligned}
& x_{k, 0}=x_{k} ; \\
& \text { Para }(\mathrm{i}=1 \text { até } \mathrm{i}=\mathrm{m})\{ \\
& \quad x_{k, i}=\left(1-\lambda_{k}\right) x_{k, i-1}+\lambda_{k} \mathscr{P}_{X_{i}}\left(x_{k, i-1}\right) \\
& \} \\
& x_{k+1}=x_{k, m}
\end{aligned}
$$$$
\} \text {. }
$$

Do mesmo modo, quando o conjunto de solução de (3.3) é vazio, podemos definir $g(x):=\frac{1}{2} \sum_{i=1}^{n} d_{X_{i}}^{2}(x)$, 
$r(x):=c, X:=\mathbb{R}^{n}$ e obter convergência quando $\lambda_{k} \rightarrow 0^{+} \mathrm{e} \sum_{k=0}^{\infty} \lambda_{k}=\infty[26]$.

\subsubsection{Cimmino}

Alguns estudos foram feitos com o algoritmo POCS com o objetivo de entender a velocidade de convergência quando o conjunto solução é vazio [23]. O problema do algoritmo é a necessidade de $\lambda_{k} \rightarrow 0^{+}$, uma vez que o tamanho de $\lambda_{k}$ indica o quanto nos afastaremos do ponto atual em direção ao conjunto convexo em questão. Portanto, a medida que as iterações acontecem, os passos dados ficam cada vez menores, tornando a convergência indesejavelmente lenta.

Felizmente, o algoritmo Cimmino contorna este inconveniente [27]. Essencialmente, o algoritmo faz a cada iteração uma "média" de todas as projeções nos conjuntos e atualiza o ponto com esta informação. Em outras palavras, definindo $g(x):=\frac{1}{2} \sum_{i=1}^{n} d_{X_{i}}^{2}(x), r(x):=c$ e $X:=\mathbb{R}^{n}$, temos:

\section{Algoritmo 3 - Cimmino}

Dados: $x_{o} \in \mathbb{R}^{n} ; b \in \mathbb{R}^{m} ;\left\{\lambda_{k}\right\} \subset[\delta, 2-\delta] \operatorname{com} \delta \in(0,1)$

Enquanto (critério de parada $=$ falso $)\{$ \} .

$$
x_{k+1}=\left(1-\lambda_{k}\right) x_{k}+\lambda_{k}\left[\frac{1}{m} \sum_{i=1}^{m} \mathscr{P}_{X_{i}}\left(x_{k}\right)\right]
$$

\subsubsection{EM, OS-EM, RAMLA E BSREM}

Até este momento, apresentamos algoritmos que tinham como intenção ou resolver exatamente o sistema (3.3) ou minimizar a distância da solução aos conjuntos convexos que representam as equações do sistema. Os algoritmos EM, OS-EM, RAMLA e BSREM têm como principal objetivo considerar a probabilidade da solução $x$ ter gerado os dados representados pelo vetor $b$. Como pode ser visto em [28], o processo de emissão e detecção de radiação segue o modelo de Poisson, ou 
seja, podemos dizer que a probabilidade de obtermos $b$ dado $x$ é:

$$
P(b \mid x)=\prod_{i=1}^{m}\left[\frac{\left\langle a^{i}, x\right\rangle^{b_{i}}}{b_{i} !} \exp \left(-\left\langle a^{i}, x\right\rangle\right)\right] .
$$

Naturalmente, busca-se, nestes algoritmos, a solução que apresente a maior probabilidade. Equivalentemente, buscamos maximixar a função abaixo para $x \geq 0$ :

$$
L(x)=\sum_{i=1}^{m} b_{i} \log \left\langle a^{i}, x\right\rangle-\left\langle a^{i}, x\right\rangle \text {. }
$$

Portanto, $g(x):=-L(x)$ para todos estes algoritmos. Ainda, vale observar que, no algoritmo BSREM, existe a preocupação de regularização da imagem com alguma $r(x)$ convexa.

Não nos preocuparemos com a apresentação desses algoritmos, uma vez que os nossos anseios científicos não os envolvem de forma direta, entretanto, não poderíamos deixar de citá-los aqui. Para um conhecimento amplo dos algoritmos e de suas convergências, ver [29-32].

\subsubsection{OS-SPS}

Como os algoritmos anteriores, OS-SPS baseia-se na maximização de L(x) e como em BSREM, procura uma regularização para a imagem tomográfica com uma função $r(x)$ convexa. A convergência pode ser vista em [33,34], sendo que o algoritmo usa uma versão modificada da logverossimilhança, a qual denotaremos por $\bar{L}$. Assim, se $g(x)=-\bar{L}_{l}(x), r(x)$ convexa e $X=\mathbb{R}_{+}^{n}$, temos:

\section{Algoritmo 4 - OS-SPS}

Dados: $x_{o} \in \mathbb{R}_{++}^{n} ; b \in \mathbb{R}^{m} ; \gamma>0 ;\left\{\lambda_{k}\right\} \subset \mathbb{R}_{++} \operatorname{com} \sum_{k=0}^{\infty} \lambda_{k}=\infty$ e $\sum_{k=0}^{\infty} \lambda_{k}^{2}<\infty ; D$ uma matriz diagonal definida positiva; $B$ é um conjunto que representa restrições de caixa; $I_{1}, \ldots, I_{S}$ com $\bigcup_{i=1}^{s} I_{i}=\{1, \ldots, m\}$ e $i \neq j \Rightarrow I_{i} \cap I_{j}=\emptyset$.

Enquanto (critério de parada $=$ falso $)\{$

$$
\begin{aligned}
& x_{k, 0}=x_{k} ; \\
& \operatorname{Para}(l=1 \text { até } l=s)\{ \\
& \qquad x_{k, l}=\mathscr{P}_{B}\left(x_{k, l-1}+\lambda_{k} D\left(\nabla \bar{L}_{l}\left(x_{k, l-1}\right)-\frac{\gamma}{s} \nabla r\left(x_{k, l-1}\right)\right)\right) ;
\end{aligned}
$$


$x_{k+1}=x_{k, s}$

\} .

Apresentamos agora um método diferente dos que foram propostos até agora. Este método apoia-se na Teoria da Amostragem Compressiva e propõe a solução de um novo problema de otimização.

\subsubsection{Um Método Alternativo de Reconstrução Tomográfica}

Vamos considerar um sistema linear $R x=b$ com mais variáveis do que equações. Se supusermos que existe um operador linear $S$ tal que $S x$ é um vetor com grande esparsidade e a matriz $R S^{-1}$ satisfaz a Propriedade 6, então a teoria conhecida como Amostragem Compressiva (CS) [35-38] garante que, para um sistema sem ruído, a solução exata pode ser obtida se solucionarmos o seguinte problema de minimização:

$$
\min :\|S x\|_{1}
$$

$$
\text { sujeito a : } R x=b \text {. }
$$

Propriedade 6 (Propriedade da Isometria Restrita) Seja $A \in \mathbb{R}^{m \times n}$ e seja $s<p$ um inteiro. Assim, se existe uma constante $\delta_{s}$ tal que para toda submatriz $A_{s}$ de A de ordem $m \times s$ e para todo $y$ acontece

$$
\left(1-\delta_{S}\right)\|y\|^{2} \leq\left\|A_{s} y\right\|^{2} \leq\left(1+\delta_{s}\right)\|y\|^{2},
$$

então dizemos que a matriz A satisfaz a propriedade da isometria restrita com constante de isometria restrita $\delta_{s}$.

Para o caso em que este sistema linear é advindo de um processo de tomografia, podemos por meio de diferenças finitas da função $f$ de atenuação (uma vez que o problema encontra-se discretizado), encontrar um operador $S$ de tal modo que $S x$ seja um vetor esparso e $\|S x\|_{1}=T V(x)$, sendo

$$
T V(x):=\sum_{i=1}^{r_{2}} \sum_{j=1}^{r_{1}} \sqrt{\left(x_{i, j}-x_{i-1, j}\right)^{2}+\left(x_{i, j}-x_{i, j-1}\right)^{2}},
$$

$x_{i, j}$ o valor da taxa de atenuação no pixel $i, j$ e $r_{1}$ e $r_{2}$ as quantidades de pixeis na horizontal e vertical, respectivamente. 
Todavia, como na tomografia o vetor de dados $b$ apresenta ruído, podemos aproximar a solução do sistema linear resolvendo

$$
\min : T V(x)
$$

$$
\text { sujeito a : }\|R x-b\| \leq \varepsilon
$$

sendo $\varepsilon>0$ um número real que está relacionado com o nível de ruído introduzido no sistema, ou seja, $\varepsilon \approx\left\|b-R x^{*}\right\|$, sendo $x^{*}$ a imagem real. Vale ressaltar que não existe garantia que a matriz $R$ satisfaça as condições exigidas, entretanto, na prática, a recuperação da imagem é feita com sucesso.

Obviamente, uma grande dificuldade é encontrar $\varepsilon$ que represente de algum modo o nível de ruído, uma vez que não temos conhecimento de $x^{*}$. Deste modo, $\varepsilon$ é estimado resolvendo (3.8) várias vezes para diferentes valores de $\varepsilon$ e escolhemos a partir de uma determinada métrica 0 melhor $\varepsilon$. Claramente, a ideia de resolvermos (3.8) várias vezes implica em um aumento no custo computacional.

Para solucionarmos este problema, ao invés de encontrarmos $\varepsilon$, queremos encontrar em qual iteração é desejável que o algoritmo pare para que o vetor $x_{k}$ obtido seja o mais próximo da imagem real possível. Assim, uma forma natural de fazermos isto é resolver

$$
\min : T V(x)
$$

$$
\text { sujeito a : } x \in X \text {, }
$$

sendo $X:=\left\{x \in \mathbb{R}_{+}^{n} \mid\|R x-b\|_{2}^{2} \leq\|R z-b\|_{2}^{2}, \forall z \in \mathbb{R}^{n}\right\}$. Logo, aplicaremos o algoritmo desenvolvido neste estudo para resolver o problema proposto acima, com a finalidade de recuperar uma imagem conhecida na literatura como Shepp-Logan Phantom [39]. 


\section{Resultados Numéricos}

Neste capítulo apresentamos os resultados numéricos obtidos pela aplicação dos nossos métodos em dois problemas distintos. Primeiro resolvemos um problema idealizado de apenas 2 variáveis com o objetivo de ilustrar o comportamento de ambos os algoritmos, e posteriormente, para mostrar que nossos métodos são viáveis, resolvemos um problema de tomografia que envolve um total de 65536 variáveis.

\subsection{Problema Idealizado}

Sejam $x^{*}=\left[\begin{array}{ll}19.5 & 42\end{array}\right]^{T}$ e $f, g: \mathbb{R}^{2} \rightarrow \mathbb{R}$ funções convexas tais que

$$
f(x)=\sum_{i=1}^{20}\left\|R_{i} x-R_{i} x^{*}-s_{i}\right\|_{1},
$$

sendo $R_{i} \in \mathbb{R}^{2 \times 2}$ e $s_{i} \in \mathbb{R}^{2}$ respectivamente matrizes e vetores aleatórios que seguem a distribuição normal e

$$
g(x)=\sum_{i=1}^{10} g_{i}(x)=\sum_{i=1}^{10}\left(\max \left\{70, a_{1 i} e^{x_{1}}+b_{1 i} x_{2}\right\}+\max \left\{50, a_{2 i} x_{1}+b_{2 i} x_{2}^{2}\right\}\right),
$$

sendo $0<a_{1 i}<1$ e $0<a_{2 i}, b_{1 i}, b_{2 i}<10$ escalares gerados randomicamente pela distribuição uniforme. Desta forma, o problema idealizado é definido pelo seguinte problema de otimização convexa:

$$
\begin{aligned}
& \text { Min: } f(x) \\
& \text { s.a: } x \in \underset{x \in C}{\arg \min } g(x),
\end{aligned}
$$

sendo $C:=\left\{x \in \mathbb{R}^{2} \mid \phi(x) \leq 0\right\}, \operatorname{com} \phi(x)=18\left(x_{1}-3.5\right)^{2}+2\left(x_{2}-2\right)^{2}-4.5$. Ressaltamos que este último conjunto satisfaz a Condição de Slater, e portanto, ambos os algoritmos podem ser aplicados 
com sucesso ao problema proposto. Desta forma, definindo

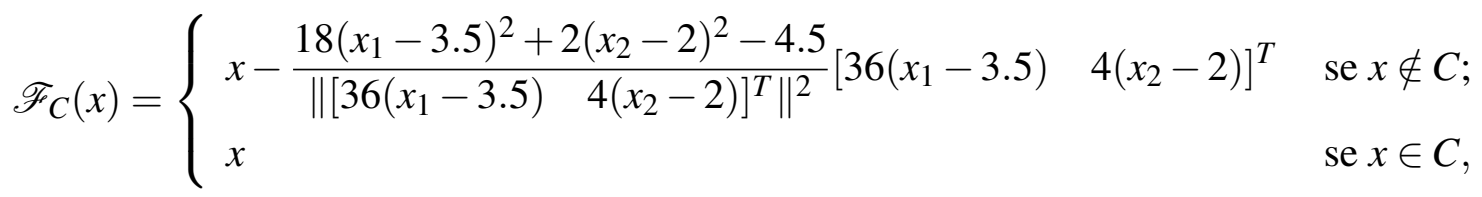

$$
\begin{aligned}
& \mathscr{F}_{X}\left(\mu_{k}, x\right)= \begin{cases}\mathscr{O}_{\phi}\left(\mu_{k}, x\right) & \text { se } x \notin C \\
\mathscr{O}_{g}\left(\mu_{k}, x\right) & \text { se } x \in C\end{cases}
\end{aligned}
$$

e $l$ o menor inteiro tal que $\mathscr{F}_{C}^{l}\left(x_{k+2 / 3}\right) \leq 100 \mu_{k}$, as seguintes iterações podem ser utilizadas para os métodos desenvolvidos:

- Algoritmo I

$$
\begin{aligned}
x_{k+1 / 3} & =\mathscr{O}_{f}\left(\lambda_{k}, x_{k}\right) \\
x_{k+2 / 3} & =\mathscr{O}_{g}\left(\mu_{k}, x_{k+1 / 3}\right) \\
x_{k+1} & =\mathscr{F}_{C}^{l}\left(x_{k+2 / 3}\right),
\end{aligned}
$$

- Algoritmo II

$$
\begin{aligned}
x_{k+1 / 2} & =\mathscr{O}_{f}\left(\lambda_{k}, x_{k}\right) \\
x_{k+1} & =\mathscr{F}_{X}\left(\mu_{k}, x_{k+1 / 2}\right) .
\end{aligned}
$$

Para ambos os algoritmos, o método de subgradiente incremental foi utilizado para todos os operadores de otimalidade (exceto para $\mathscr{O}_{\phi}$, no qual foi empregado o método do subgradiente) juntamente com os seguintes subgradientes:

$$
\begin{aligned}
& \tilde{\nabla} g_{i}(x)=\left\{\begin{array}{cl}
{\left[\begin{array}{ll}
0 & 0
\end{array}\right]^{T}} & \text { se } a_{2 i} x_{1}+b_{2 i} x_{2}^{2} \leq 50 \text { e } a_{1 i} e^{x_{1}}+b_{1 i} x_{2} \leq 70 \\
{\left[\begin{array}{cc}
a_{2 i} & 2 b_{2 i} x_{2}
\end{array}\right]^{T}} & \text { se } a_{2 i} x_{1}+b_{2 i} x_{2}^{2}>50 \text { e } a_{1 i} e^{x_{1}}+b_{1 i} x_{2} \leq 70 \\
{\left[\begin{array}{ll}
a_{1 i} e^{x_{1}} & b_{1 i}
\end{array}\right]^{T}} & \text { se } a_{2 i} x_{1}+b_{2 i} x_{2}^{2} \leq 50 \text { e } a_{1 i} e^{x_{1}}+b_{1 i} x_{2}>70 \\
{\left[\begin{array}{ll}
a_{1 i} e^{x_{1}}+a_{2 i} & b_{1 i}+2 b_{2 i} x_{2}
\end{array}\right]^{T}} & \text { se } a_{2 i} x_{1}+b_{2 i} x_{2}^{2}>50 \text { e } a_{1 i} e^{x_{1}}+b_{1 i} x_{2}>70
\end{array}\right. \\
& \tilde{\nabla} f_{i}(x)=R_{i}^{T} \operatorname{sign}\left(R_{i} x-R_{i} x^{*}-s_{i}\right) ; \\
& \tilde{\nabla} \phi(x)=\left[\begin{array}{ll}
36\left(x_{1}-3.5\right) & 4\left(x_{2}-2\right)
\end{array}\right]^{T} \text {. }
\end{aligned}
$$

Com o intuito de estudarmos a interferência do tamanho de passo na convergência de ambos os métodos, alteramos os parâmetros $\sigma, \kappa, \alpha_{1}, \alpha_{2} \mathrm{e}\|\cdot\|_{\delta}$ apresentados nas regras de tamanho de passo abaixo e geramos as Tabelas 4.1 e 4.2, as quais representam, para cada conjunto de parâmetros, o erro da milésima iteração com o ponto ótimo $x^{*}$ do problema. Vale salientar que o ponto ótimo utilizado para estas comparações foi obtido de forma analítica, uma vez que a representação do problema em um espaço bidimensional permitiu que soubéssemos que este ponto pode ser encon- 
trado interseccionando a fronteira do conjunto $C$ com a fronteira do conjunto formado pelos pontos de mínimo da função $g$ em $\mathbb{R}^{2}$, obtendo $x^{*}=\left[\begin{array}{ll}3.924737732689 & 1.208557818860\end{array}\right]^{T}$.

- Tamanhos de passo do Algoritmo I

$$
\begin{gathered}
\lambda_{0}=\frac{\sigma}{\kappa\left\|\tilde{\nabla} f\left(x_{0}\right)\right\|_{\delta}} \quad \text { e } \quad \lambda_{k}=\lambda_{0} \frac{\left(1+0.999 c_{k}\right)}{k^{\alpha_{1}}} \\
\mu_{0}=\frac{\sigma}{\kappa\left\|\tilde{\nabla} g\left(x_{0}\right)\right\|_{\delta}} \quad \text { e } \quad \mu_{k}=\mu_{0} \frac{1}{k^{\alpha_{2}}},
\end{gathered}
$$

- Tamanhos de passo do Algoritmo II

$$
\begin{gathered}
\lambda_{0}=\frac{\sigma}{\kappa\left\|\tilde{\nabla} f\left(x_{0}\right)\right\|_{\delta}} \quad \text { e } \quad \lambda_{k}=\lambda_{0} \frac{\left(1+0.999 c_{k}\right)}{k^{\alpha_{1}}} \\
\mu_{0}=\frac{\sigma}{\frac{1}{2} \kappa\left\|\tilde{\nabla} g\left(x_{0}\right)\right\|_{\delta}+\frac{1}{2} \kappa\left\|\tilde{\nabla} \phi\left(x_{0}\right)\right\|_{\delta}} \quad \text { e } \quad \mu_{k}=\mu_{0} \frac{1}{k^{\alpha_{2}}}
\end{gathered}
$$

sendo $x_{0}=(3.5,4.0)$,

$$
c_{k}=\frac{\left\langle x_{k-2 / 3}-x_{k-1}, x_{k}-x_{k-2 / 3}\right\rangle}{\left\|x_{k-2 / 3}-x_{k-1}\right\|\left\|x_{k}-x_{k-2 / 3}\right\|} \quad \text { e } \quad d_{k}=\frac{\left\langle x_{k-1 / 2}-x_{k-1}, x_{k}-x_{k-1 / 2}\right\rangle}{\left\|x_{k-1 / 2}-x_{k-1}\right\|\left\|x_{k}-x_{k-1 / 2}\right\|} .
$$

Observemos que os valores $c_{k}$ e $d_{k}$ nada mais são que os ângulos formados entre as direções de otimalidade e as direções de factibilidade tomadas pelos algoritmos em cada iteração, e desta forma, as parcelas $\left(1+0.999 c_{k}\right)$ e $\left(1+0.999 d_{k}\right)$ tendem a favorecer a direção de factibilidade durante cada iteração. Ademais, definimos $\mu_{0}$ no Algoritmo II fazendo uso da média dos subgradientes das funções $g$ e $\phi$ no ponto $x_{0}$, pois entendemos que esta é a melhor forma de normalizarmos o passo tomado na direção de factibilidade, uma vez que no Algoritmo II, o operador factível é definido ora pelo operador de otimalidade de $g$, ora pelo operador de $\phi$.

\begin{tabular}{|c|c|c|c|c|}
\hline$\left(\alpha_{1}, \alpha_{2}\right)$ & $(1,0.75)$ & $(1,0.51)$ & $(0.75,0.51)$ & $(0.75,0.38)$ \\
\hline$\left(\sigma, \kappa,\|\cdot\|_{\delta}\right)$ & $1.3 \cdot 10^{-1}$ & $8.2 \cdot 10^{-4}$ & $8.7 \cdot 10^{-4}$ & $5.7 \cdot 10^{-4}$ \\
\hline$\left(1,1,\|\cdot\|_{2}\right)$ & $7.1 \cdot 10^{-4}$ & $4.9 \cdot 10^{-3}$ & $9.8 \cdot 10^{-3}$ & $7.3 \cdot 10^{-3}$ \\
\hline$\left(\left\|x_{0}\right\|, 1,\|\cdot\|_{2}\right)$ & $2.1 \cdot 10^{-1}$ & $1.0 \cdot 10^{-1}$ & $1.5 \cdot 10^{-3}$ & $3.7 \cdot 10^{-4}$ \\
\hline$\left(1, n,\|\cdot\|_{\infty}\right)$ & $1.3 \cdot 10^{-3}$ & $6.9 \cdot 10^{-4}$ & $7.6 \cdot 10^{-3}$ & $5.0 \cdot 10^{-3}$ \\
\hline$\left(\left\|x_{0}\right\|, n,\|\cdot\|_{\infty}\right)$ &
\end{tabular}

Tabela 4.1: Para cada conjunto de parâmetros a tabela exibe a ordem de erro da milésima iteração do Algoritmo I com o ponto ótimo $x^{*}$ do problema, i.e, $\left\|x_{1000}-x^{*}\right\|_{\infty}$. Na tabela, a letra $n$ representa o número de variáveis do problema, neste caso, $n=2$.

Observando as duas tabelas expostas anteriormente, podemos notar que ambos os algoritmos apresentam melhores soluções quando os valores de $\alpha_{1}$ e $\alpha_{2}$ estão mais distantes e que o uso da norma euclidiana ou da norma do máximo não acarretam diferenças claras nos pontos encontrados. 


\begin{tabular}{|c|c|c|c|c|}
\hline$\left(\alpha_{1}, \alpha_{2}\right)$ & $(1,0.75)$ & $(1,0.51)$ & $(0.75,0.51)$ & $(0.75,0.38)$ \\
\hline$\left(\sigma, \kappa,\|\cdot\|_{\delta}\right)$ & $2.3 \cdot 10^{0}$ & $2.6 \cdot 10^{-1}$ & $3.9 \cdot 10^{-1}$ & $4.7 \cdot 10^{-3}$ \\
\hline$\left(1,1,\|\cdot\|_{2}\right)$ & $9.9 \cdot 10^{-2}$ & $6.2 \cdot 10^{-3}$ & $6.2 \cdot 10^{-2}$ & $2.4 \cdot 10^{-2}$ \\
\hline$\left(\left\|x_{0}\right\|, 1,\|\cdot\|_{2}\right)$ & $2.2 \cdot 10^{0}$ & $4.3 \cdot 10^{-1}$ & $7.3 \cdot 10^{-1}$ & $3.0 \cdot 10^{-2}$ \\
\hline$\left(1, n,\|\cdot\|_{\infty}\right)$ & $7.4 \cdot 10^{-1}$ & $4.7 \cdot 10^{-3}$ & $3.5 \cdot 10^{-1}$ & $1.4 \cdot 10^{-2}$ \\
\hline$\left(\left\|x_{0}\right\|, n,\|\cdot\|_{\infty}\right)$ &
\end{tabular}

Tabela 4.2: Para cada conjunto de parâmetros a tabela exibe a ordem de erro da milésima iteração do Algoritmo II com o ponto ótimo $x^{*}$ do problema, i.e, $\left\|x_{1000}-x^{*}\right\|_{\infty}$. Na tabela, a letra $n$ representa o número de variáveis do problema, neste caso, $n=2$.

Assim, para que o estudo destes algoritmos seja mais ilustrativo, geramos as Figuras 4.1 e 4.2 com os parâmetros $\left(\alpha_{1}, \alpha_{2}, \sigma, \kappa,\|\cdot\|_{\delta}\right)=\left(0.75,0.38,1,1,\|\cdot\|_{2}\right)$.
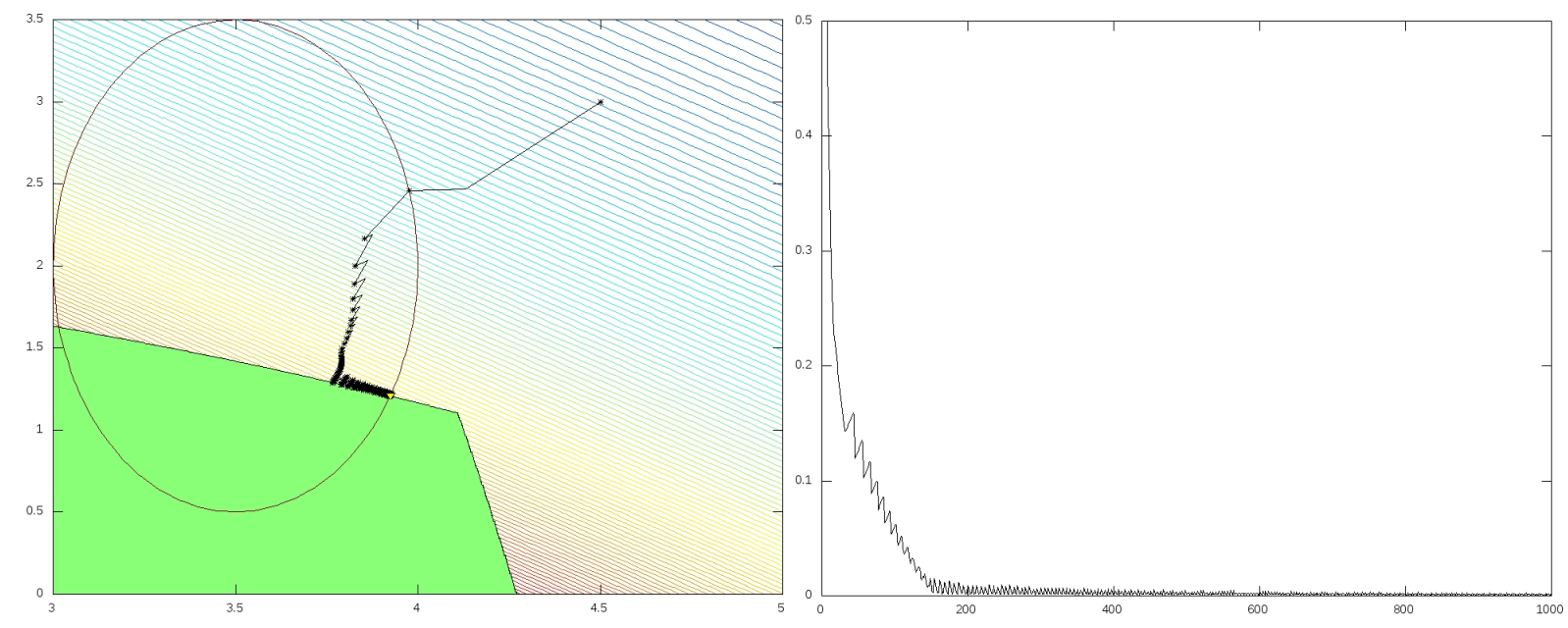

Figura 4.1: A imagem da esquerda ilustra a representação do espaço factível juntamente com as iterações do Algoritmo I. O plano de fundo hachurado representa as curvas de nível da função $f$, a região verde clara determina os pontos de mínimo da função $g \mathrm{em} \mathbb{R}^{2}$, a elipse em vermelho delimita o conjunto $C$, e o triângulo amarelo indica a última iteração, ou seja, $x_{1000}$. Já a imagem da direita ilustra a distância das iterações à solução ótima $x^{*}$ do problema, sendo o eixo horizontal o número de iterações e o vertical a distância $\left\|x_{k}-x^{*}\right\|_{\infty}$.

Apesar de ser apenas um único experimento, podemos tirar algumas informações importantes de ambos os algoritmos enquanto analisamos as imagens obtidas. Notemos primeiramente que nas primeiras iterações do Algoritmo II o método tende a se aproximar do ponto mínimo de $f$ em $C$ e somente quando as iterações adentram no conjunto $C$ que ocorre a preferência pela solução ótima verdadeira. Isto já era esperado, uma vez que quando os iterandos não encontram-se em $C$ os únicos operadores que são aplicados são os operadores que minimizam $f$ e a função $\phi$, ou seja, durante estas iterações o método não usa nenhuma informação sobre a função $g$. Consequentemente, podemos inferir que se o ponto mínimo de $f$ em $C$ está próximo da solução ótima original de um determinado problema, o Algoritmo II tem a tendência de encontrar a solução mais rapidamente que o Algoritmo I, enquanto que se o ponto mínimo de $f$ em $C$ está distante da solução original, o 

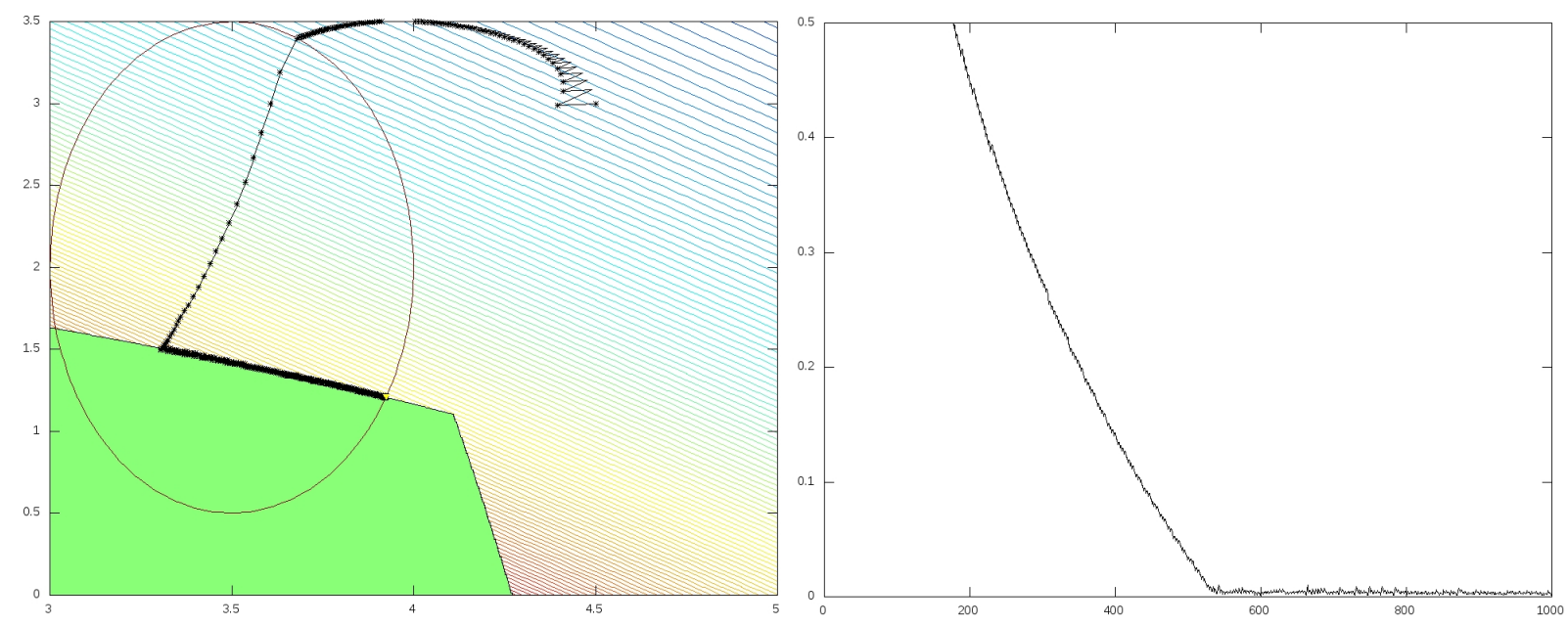

Figura 4.2: A imagem da esquerda ilustra a representação do espaço factível juntamente com as iterações do Algoritmo II. O plano de fundo hachurado representa as curvas de nível da função $f$, a região verde clara determina os pontos de mínimo da função $g$ em $\mathbb{R}^{2}$, a elipse em vermelho delimita o conjunto $C$ e o triângulo amarelo indica a última iteração, ou seja, $x_{1000}$. Já a imagem da direita ilustra a distância das iterações à solução ótima $x^{*}$ do problema, sendo o eixo horizontal o número de iterações e o vertical a distância $\left\|x_{k}-x^{*}\right\|_{\infty}$.

inverso ocorre. Por fim, podemos notar que próximo da solução ótima a inconstância da aplicação do operador $\mathscr{O}_{g}$ no Algoritmo II faz com que este oscile mais que o Algoritmo I.

\subsection{Problema de Recuperação de Imagem}

Como dito anteriormente, o problema de recuperação de imagens tomográficas pode ser resumido a um sistema linear $(R x=b)$. Infelizmente, a solução deste sistema não é trivial, uma vez que o problema é mal-posto e a emissão-detecção de partículas radioativas segue a distribuição de Poisson, ocasionando um ruído indesejado na imagem recuperada. Assim, com o intuito de obter um método de recuperação de imagem alternativo, decidimos resolver o seguinte problema de otimização:

$$
\begin{aligned}
& \text { Min: } \operatorname{TV}(x) \\
& \text { s.a: } x \in \arg \min _{x \in \mathbb{R}_{+}^{n}} g(x)
\end{aligned}
$$

sendo a função $T V(x)$ definida como em (3.7) e $g(x)=\|R x-b\|^{2}$.

Como imagem original $\left(x^{*}\right)$ utilizada para o experimento, fizemos uso da figura Shepp-Logan Head Phantom discretizada em $256 \times 256$ pixels, proporcionando um problema de 65536 variáveis. Além disso, para gerarmos o vetor $b$ que representa os dados obtidos pelo tomógrafo, fizemos $b_{i} \sim \operatorname{Poisson}\left[\left(R x^{*}\right)_{i}\right]$, alcançando uma taxa de ruído de $\left\|R x^{*}-b\right\| /\|b\| \approx 5.6 \%$. 
Observemos que neste experimento não há diferença no comportamento dos métodos desenvolvidos no Capítulo 2, sendo ambos, essencialmente, o mesmo algoritmo, uma vez que o conjunto $C$ admite uma projeção simples. Desta forma, fizemos uso das seguintes iterações para a encontrarmos a solução do problema proposto:

$$
\begin{aligned}
x_{k+1 / 3} & =x_{k}-\lambda_{k} \tilde{\nabla} T V\left(x_{k}\right) \\
x_{k+2 / 3} & =\mathscr{O}_{g}\left(\mu_{k}, x_{k+1 / 3}\right) \\
x_{k+1} & =\mathscr{P}_{\mathscr{R}_{+}^{n}}\left(x_{k+2 / 3}\right) .
\end{aligned}
$$

Durante a execução do algoritmo foi utilizado o método de subgradiente incremental como operador de otimalidade da função $g$, separando-a em 10 sub-funções. Ainda, adotando como figura inicial o vetor constante $x_{0}=\alpha(1, \ldots, 1)$, com $\alpha$ satisfazendo $\sum_{i}\left(R x_{0}\right)_{i}=\sum_{i} b_{i}$, definimos os tamanhos de passos como

$$
\omega=\frac{\left\|x_{0}\right\|}{\left\|\tilde{\nabla} g\left(x_{0}\right)\right\|}, \quad \lambda_{k}=\frac{\omega}{(k+1)^{0.5}}, \quad \mu_{k}=\frac{\omega}{(k+1)^{0.45}},
$$

seguidos pelo subsequente subgradiente da função $T V$ :

$$
\begin{aligned}
(\tilde{\nabla} T V(x))_{i, j}= & \frac{2 x_{i, j}-x_{i, j-1}-x_{i-1, j}}{\sqrt{\left(x_{i, j}-x_{i, j-1}\right)^{2}+\left(x_{i, j}-x i-1, j\right)^{2}}} \\
& +\frac{x_{i, j}-x_{i, j+1}}{\sqrt{\left(x_{i, j+1}-x_{i, j}\right)^{2}+\left(x_{i, j+1}-x i-1, j+1\right)^{2}}} \\
& +\frac{x_{i, j}-x_{i+1, j}}{\sqrt{\left(x_{i+1, j}-x_{i, j}\right)^{2}+\left(x_{i+1, j}-x i+1, j-1\right)^{2}}},
\end{aligned}
$$

sendo que quando algum dos denominadores de $(\tilde{\nabla} T V(x))_{i, j}$ é nulo, a correspondente parcela é zerada.

Diferentemente do problema idealizado, a limitação do conjunto $X$ deste problema não é de verificação imediata. Uma saída para este inconveniente, sem de fato demonstrar a limitação deste conjunto, é adicionar uma projeção a um conjunto limitado ao final do algoritmo, uma vez que pixels com valores altos não possuem sentido físico. Todavia, isto não foi necessário, pois durante a execução do algoritmo 4.1, notamos que a sequência gerada era limitada, e desta forma, pelo Teorema 2.2, a convergência da sequência foi assegurada.

Pela discussão feita no Capítulo 3, sabemos que os minimizadores da função $g$ possuem uma quantidade significativa de ruído, produzindo uma imagem de baixa qualidade. Por este motivo, os expoentes utilizados nos tamanhos de passo $\lambda_{k}$ e $\mu_{k}$ são próximos, uma vez que não queremos que nas primeiras iterações o algoritmo nos leve rapidamente para os minimizadores da função g. Além disso, graças à característica de rápida convergência nas primeiras iterações de métodos que envolvem subgradientes, obtemos imagens úteis logo nas iterações iniciais, permitindo que a 
execução do algoritmo fosse cessada rapidamente. Assim, com apenas 100 iterações e com 8192 medidas, a imagem foi reconstruída em 8.6 segundos em um computador Intel Core 2 Duo CPU T6500, demonstrando o baixo custo computacional das iterações.
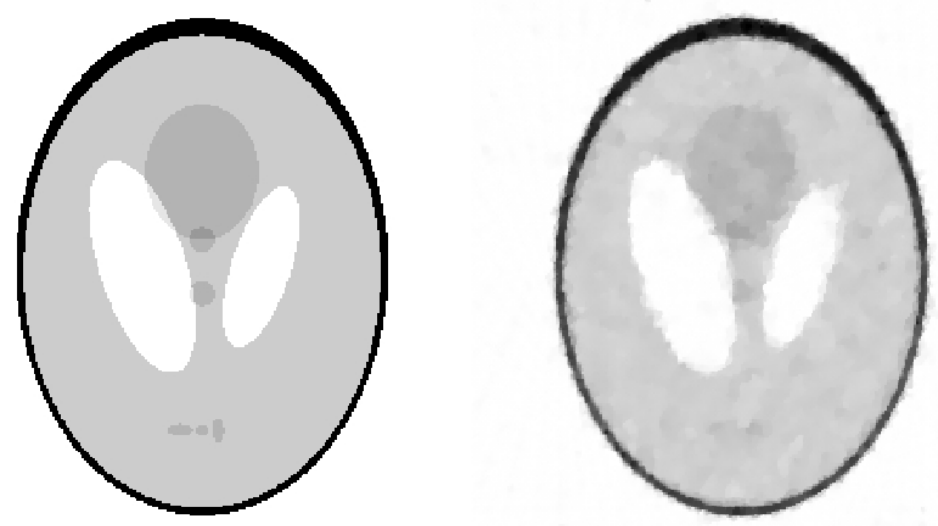

Figura 4.3: A esquerda temos a imagem original e a direita é apresentada a imagem reconstruída pelo nosso algoritmo com apenas 100 iterações.

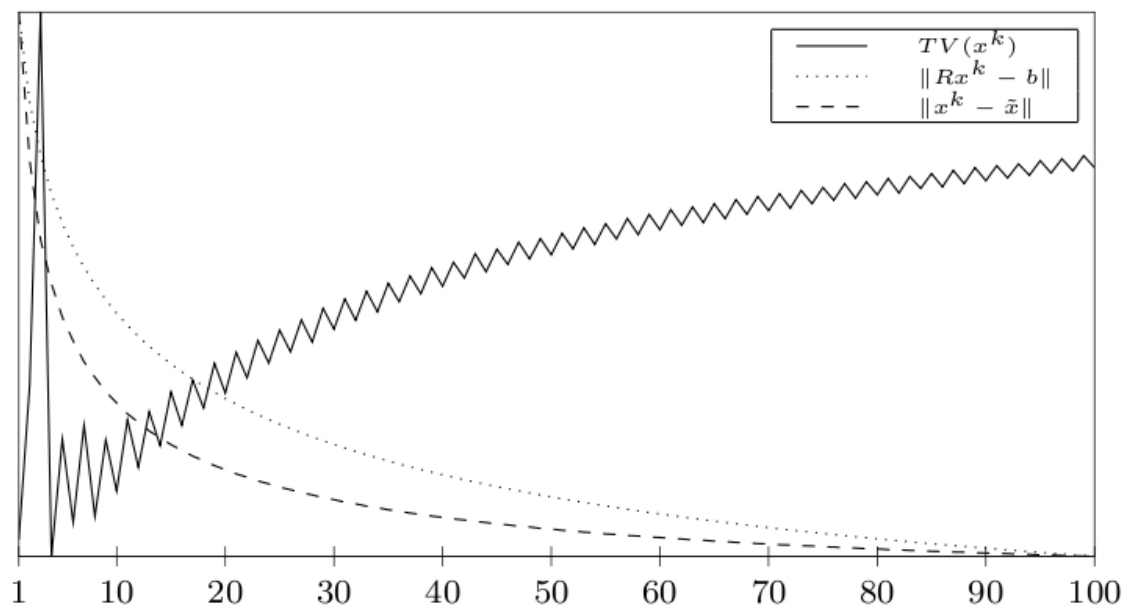

Figura 4.4: Evolução dos valores normalizados das parcelas $\left\|x_{k}-x^{*}\right\|,\left\|R x_{k}-b\right\|$ e $T V\left(x_{k}\right)$ em cada iteração.

A Figura 4.4 mostra a evolução do valor das funções $T V$ e $g$ ao longo das iterações, bem como a distância para a imagem original. A oscilação da curva $T V$ pode ser removida se redimensionarmos o tamanho de passo $\mu_{k}$ para um valor apropriado, contudo, decidimos não estragar o experimento reescalando em demasia os parâmetros para uma única instância. Por outro lado, as demais curvas apresentadas, mostram a aproximação das iterações para a imagem ideal, o que pode ser confirmado pela Figura 4.3. Desta forma, foi possível reconstruir uma imagem tomográfica resolvendo um problema inspirado na Teoria da Amostragem Compressiva e obter uma reconstrução de boa qualidade. 



\section{Conclusão}

Construímos dois novos métodos para a solução de problemas de otimização convexa em dois níveis não necessariamente diferenciáveis. Como vantagem, temos que ambos os métodos permitem a inclusão de algoritmos incrementais em seus operadores e mostramos que em nenhum momento é necessária a solução de subproblemas para que os métodos convirjam, uma vez que não exigimos direções de descida para o funcionamento dos algoritmos. Também mostramos que a utilização de projeções sobre o conjunto $C$ não se faz necessária quando estas são caras computacionalmente.

Para demonstrar a viabilidade das ferramentas desenvolvidas, solucionamos um problema de reconstrução de imagens tomográficas, obtendo um método competitivo com o estado da arte, dada a rapidez das iterações e a qualidade da imagem reconstruída.

Finalmente, esta pesquisa sugere como um trabalho futuro, comparações extensivas com métodos já existentes, bem como a real influência dos tamanhos de passo na velocidade de convergência destes algoritmos. Ademais, estes métodos podem representar um ponto de partida na elaboração de algoritmos para a solução de problemas de otimização em dois níveis com a função custo não satisfazendo a condição de convexidade. 


\section{Bibliografia}

[1] JEAN-BAPTISTE; HIRIART-URRUTY; LEMARECHAL, C. Convex analysis and minimization algorithms. Springer, 1996. v. 1.

[2] SHOR, N.; KIWIEL, K.; RUSZCZYNSKI, A. Minimization methods for non-differentiable functions. Springer-Verlag Berlin, 1985.

[3] SOLODOV, M. V.; ZAVRIEV, S. K. Error stability properties of generalized gradient-type algorithms. Journal of Optimization Theory and Applications, v. 98, p. 663-680, 1998.

[4] NEDIC, A.; BERTSEKAS, D. Incremental subgradient methods for nondifferentiable optimization. SIAM Journal on Optimization, v. 12, n. 1, p. 109-138, 2001.

[5] BLATT, D.; HERO, A. O.; GAUCHMAN, H. A convergent incremental gradient method with a constant step size. SIAM Journal on Optimization, v. 18, p. 29-51, 2007.

[6] BERTSEKAS, D. Incremental proximal methods for large scale convex optimization. Springer, 2011. v. 129.

[7] POLYAK, B. A general method for solving extremal problems. Dokl. Akad. Nauk SSSR, v. 174, p. 33-36, 1967.

[8] HELOU, E. S.; DE PIERRO, A. R. Incremental subgradients for constrained convex optimization: a unified framework and new methods. SIAM J. Optim, v. 20, p. 1547-1572, 2009.

[9] HELOU, E. S.; DE PIERRO, A. R. On perturbed steepest descent methods with inexact line search for bilevel convex optimization. Optimization, v. 60, p. 991-1008, 2011.

[10] SOLODOV, M. An explicit descent method for bilevel convex optimization. Journal of Convex Analysis, v. 14, n. 2, p. 227, 2007.

[11] SOLODOV, M. A bundle method for a class of bilevel nonsmooth convex minimization problems. SIAM Journal on Optimization, v. 18, n. 1, p. 242-259, 2008.

[12] BONNANS, J.; GILBERT, J.; LEMARÉCHAL, C.; SAGASTIZÁBAL, C. Numerical optimization: theoretical and practical aspects. Springer, 2006.

[13] WIJSMAN, R. Convergence of sequences of convex sets, cones and functions. ii. Transactions of the American Mathematical Society, p. 32-45, 1966.

[14] BERTSEKAS, D.; NEDIć, A.; OZDAGLAR, A. et al. Convex analysis and optimization. Athena Scientific, 2003.

[15] HOFFMANN, A. The distance to the intersection of two convex sets expressed by the distances to each of them. Mathematische Nachrichten, v. 157, n. 1, p. 81-98, 1992. 
[16] NATTERER, F. The mathematics of computerized tomography. Society for Industrial Mathematics, 2001. v. 32.

[17] DE PIERRO, A. Problemas matemáticos em tomografia por emissão. Matemática Universitária, , n. 41, p. 33-49, 2006.

[18] IUSEM, A. N.; DE PIERRO, A. Fundamentos matemáticos da tomografia computadorizada: Métodos de expansão em séries. Matemática Universitária, , n. 12, p. 57-85, 2006.

[19] ENGL, H. W.; HANK, M.; NEUBAUER, A. Regularization of inverse problems. Kluwer Academic Publishers, 2000.

[20] HELOU, E. A new parameter choice rule for optimization models for inverse problems with poisson noise. CMAC-SE, Anais do I Congresso de Matemática Aplicada e Computacional da Região Sudeste, 2012.

[21] GORDON, R.; BENDER, R.; HERMAN, G. T. Algebraic reconstruction techniques (ART) for three-dimensional electron microscopy and x-ray photography. Journal of Theorical Bio$\log y$, v. 29, p. 471-482, 1970.

[22] TRUMMER, M. R. Reconstructing pictures from projections: on the convergence of the art algorithm with relaxation. Computing, v. 26, p. 189-195, 1981.

[23] CENSOR, Y.; EGGERMONT, P. P. B.; GORDON, D. Strong underrelaxation in Kaczmarz's method for inconsistent systems. Numerische Mathematik, v. 41, p. 83 - 92, 1983.

[24] TRUMMER, M. R. A note on the art of relaxation. Computing, v. 33, p. 349-352, 1984.

[25] GUBIN, L.; POLYAK, B.; RAIK, E. The method of projections for finding the common point of convex sets. USSR Computational Mathematics and Mathematical Physics, v. 7, p. 1-24, 1966.

[26] CENSOR, Y.; PIERRO, A. R. D.; ZAKNOON, M. Steered sequential projections for the inconsistent convex feasibility problem. Nonlinear Analysis: Theory, Methods \& Applications, v. 59, n. 3 , p. $385-405,2004$.

[27] COMBETTES, P. L. Inconsistent signal feasibility problems: Least-squares solutions in a product space. IEEE Transactions on Signal Processing, v. 42, p. 2955-2966, 1994.

[28] HERMAN, G. T. Image reconstruction from projections: The fundamentals of computerized tomography. New York: Academic, 1980.

[29] Y. VARDI, L. A. S.; KAUFMAN, L. A statistical model for positron emission tomography. Journal of the American Statistical Association, v. 80, p. 8-20, 1985.

[30] HUDSON, H. M.; LARKIN, R. S. Accelerated image reconstruction using ordered subsets of projection data. IEEE Transactions on Medical Imaging, v. 13, p. 601-609, 1994.

[31] BROWNE, J.; ÁLVARO R. DE PIERRO. A row-action alternative to the EM algorithm for maximizing likelihoods in emission tomography. IEEE Transactions on Medical Imaging, v. 15, p. 687-699, 1996. 
[32] ÁlVARO R. DE PIERRO; YAMAGISHI, M. E. B. Fast em-likemethods for maximum “a posteriori" estimates in emission tomography. IEEE Transactions on Medical Imaging, v. 20, p. 280-288, 2001.

[33] ERDOGAN, H.; FESSLER, J. A. Ordered subsets algorithms for transmission tomography. Phys. Med. Biol., v. 44, p. 2835-2851, 1999.

[34] AHN, S.; FESSLER, J. A. Globally convergent image reconstruction for emission tomography using relaxed ordered subsets algorithms. IEEE Transactions on Medical Imaging, v. 22, p. 613-626, 2003.

[35] CANDÈS, E. J.; TAO., T. Decoding by linear programming. IEEE Transactions on Information Theory, v. 51, p. 4203-4215, 2005.

[36] CANDÈS, E. J.; TAO., T. Near-optimal signal recovery from random projections: Universal encoding strategies? IEEE Transactions on Information Theory, v. 52, p. 5406-5425, 2006.

[37] CANDÈS, E. J.; ROMBERG, J. K.; TAO, T. Robust uncertainty principles: Exact signal reconstruction from highly incomplete frequency information. IEEE Transactions on Information Theory, v. 52, p. 489-509, 2006.

[38] CANDÈS, E. J.; ROMBERG, J. K.; TAO, T. Stable signal recovery from incomplete and inaccurate measurements. Communications on Pure and Applied Mathematics, v. 59, p. 235-256, 2006.

[39] SHEPP, L.; LOGAN, B. The fourier reconstruction of a head section. IEEE Trans. Nucl. Sci, v. 21, n. 3, p. $21-43,1974$. 